\title{
Searching for the Higgsino-Bino sector at the LHC
}

\author{
Jia Liu, ${ }^{a}$ Navin McGinnis, ${ }^{b, c}$ Carlos E.M. Wagner ${ }^{a, c, d}$ and Xiao-Ping Wang ${ }^{c}$ \\ ${ }^{a}$ Physics Department and Enrico Fermi Institute, University of Chicago, \\ Chicago, IL 6063\%, U.S.A. \\ ${ }^{b}$ Physics Department, Indiana University, \\ Bloomington, IN 47405, U.S.A. \\ ${ }^{c}$ High Energy Physics Division, Argonne National Laboratory, \\ Argonne, IL 60439, U.S.A. \\ ${ }^{d}$ Kavli Institute for Cosmological Physics, University of Chicago, \\ Chicago, IL 6063\%, U.S.A. \\ E-mail: liuj1@uchicago.edu, nmmcginn@indiana.edu, cwagner@anl.gov, \\ xia.wang@anl.gov
}

ABSTRACT: We study the search for electroweakinos at the $13 \mathrm{TeV}$ LHC in the case of heavy scalar superpartners. We consider both the direct production mode and the one associated with the decay of heavy Higgs bosons, and concentrate on the case of light Higgsinos and Binos. In this case, the direct production searches becomes more challenging than in the light Wino scenario. In the direct production mode, we use the current experimental searches to set the reach for these particles at larger luminosities, and we emphasize the relevance of considering both the neutral gauge boson and the neutral Higgs decay modes of the second and third lightest neutralino. We show the complementarity of these searches with the ones induced by the decay of the heavy Higgs bosons, which are dominated by the associated production of the lightest neutralino with the second and third lightest ones, with the latter decaying into gauge bosons. We show that, depending on the value of $\tan \beta$, the Higgs boson decay channel remains competitive with the direct production channel up to heavy Higgs boson masses of about $1 \mathrm{TeV}$. Moreover, this search is not limited by the same kinematic considerations as the ones in the direct production mode and can cover masses up to the kinematic threshold for the decay of the heavier electroweakinos into the lightest neutralino. This decay mode provides also an alternative way of looking for heavy Higgs bosons in this range of masses and hence should be a high priority for future LHC analyses.

Keywords: Beyond Standard Model, Supersymmetric Standard Model

ARXIV EPRINT: 2006.07389 


\section{Contents}

1 Introduction $\quad 1$

2 Electroweakino productions 3

2.1 Direct electroweakino production 3

$\begin{array}{lll}2.2 & \text { Electroweakinos from heavy Higgs production } & 7\end{array}$

3 Reach of direct production searches of higgsino states at higher luminosities

3.1 ZW channels 9

3.2 hW channels 11

4 Reach of the Higgs decay production mode at higher luminosities 12

5 Conclusions $\quad 16$

$\begin{array}{lr}\text { A Auxiliary figures } & 17\end{array}$

$\begin{array}{ll}\text { B Results for } \operatorname{sgn}(\mu)=+1 & 17\end{array}$

\section{Introduction}

The Standard Model provides an excellent low energy effective theory describing the interaction of particles at energies similar to or smaller than the weak scale. Searches at the LHC are underway looking for the possible production of new particles with masses up to a few $\mathrm{TeV}$. No clear signal of new physics has been found, leading to strong bounds on the presence of strongly interacting particles at the $\mathrm{TeV}$ scale (see, for instance, refs. [1, 2]). However, if the particles are weakly interacting, new physics searches may be hindered by small production cross sections and large backgrounds induced by the production of the weak gauge bosons and strongly interacting states, like the top and bottom quarks. Contrary to strongly interacting states, many weakly interacting particle search channels will become statistically significant only at high luminosities. For this reason, the LHC experimental collaborations are now starting to put stronger emphasis on the search for weakly interacting particles [3-13].

Among the many hypothetical weakly interacting particles, the electroweakinos, namely the superpartners of the Higgs and electroweak gauge bosons, are well motivated examples [14-16]. They are a natural consequence of supersymmetry at the TeV scale [1721]. Weakly interacting supersymmetric particles are not subject to strong renormalization group effects in the evolution of their masses from high scales, and are therefore expected 
to be lighter than strongly interacting ones. Moreover, this sector includes the Dark Matter candidates within these theories $[22,23]$. Since Dark Matter is arguably one of the strongest reasons to expect new physics among the Standard Model, the search for these particles is particularly well justified. The superpartner of the hypercharge gauge boson, the Bino, places a special role beyond these particles, since it does not couple to any of the electroweak gauge bosons and therefore, after mixing with the heavier superpartners of the Higgs and the electroweak gauge bosons, can acquire the proper relic density for masses significantly lower than the TeV scale. The superpartners of the Higgs bosons, the Higgsinos, are very relevant in defining the light Dark Matter candidate interactions, since they mix with the Binos through the Higgs vacuum expectation values. In this work, we shall assume, for simplicity, that the superpartners of the weak gauge bosons, the Winos, are significantly heavier than the two neutral Higgsino states, which therefore will become the second and third lightest neutralinos. Moreover, we shall assume that all scalar superpartners are sufficiently heavy, so that they do not participate in a relevant way in the decays of the Higgs bosons or electroweakinos.

Searches for electroweakinos at the LHC have so far been performed in direct production channels [3-6, 8-13]. The main production channel is mediated by charged and neutral electroweak gauge bosons, and can lead to hadronic, semi-leptonic and purely leptonic final states plus missing energy. Of particular relevance among these channels is the associated production of charged and neutral electroweakino states, with charged states decaying into $W^{ \pm}$bosons and the lightest neutralino state, and the second (and third) lightest neutralino states decaying into neutral gauge and Higgs bosons. The assumption of heavy Winos and scalar superpartners tend to weaken the reach of the LHC, since Winos have higher cross sections than the Higgsinos and light sleptons tend to contribute to the electroweakino decays increasing the total branching ratio into leptons, which are easier to distinguish from the large QCD background. Since the LHC limits are usually presented considering Wino direct production, e.g. [10], we shall recast these searches by studying the Higgsino cross sections and the proper decay branching ratios into SM Higgs and $Z$-boson final states.

Although well motivated, searches for electroweakinos proceeding from the decay of heavy Higgs bosons are in a preliminary state. There has so far been only a few relevant theoretical analyses of heavy Higgs bosons decaying into electroweakino states [24-43], concentrating mostly on the associated production of the lightest, and second and third lightest neutralinos, which then lead mostly to decay channels involving single production of the $Z$ and Higgs bosons plus missing energy. Similar studies have been performed in the Next-to-Minimal-Supersymmetric-Standard-Model (NMSSM) [44-50]. These analyses show the relevance and the promising reach of these channels and serve as motivation for our work. For the comparison with the direct production channel, it is most relevant to understand the reach in the electroweakino mass parameter space, something not provided by these analyses.

In this work, we shall analyze the complementarity of the direct production and Higgs boson decay channels. The work is organized as follows: section 2 provides an analysis of the electroweakino and Higgs production cross sections. Section 3 provides the recast of existing searches to higgsino-like electroweakinos. In section 4, we concentrate on the 
di-lepton plus missing energy channel for electroweakinos from the decay of heavy neutral Higgses and then present the complementarity of this search channel with the projected bounds for direct production. Section 5 is reserved for our conclusions.

\section{Electroweakino productions}

Scenarios where all strongly-interacting superpartners are decoupled from the rest of the MSSM are particularly well motivated both from the current status of direct searches at the LHC and the measurement of the Higgs boson mass. Indeed, within the MSSM, a $125 \mathrm{GeV}$ SM-like Higgs boson mass may only be obtained for masses of the superpartners of the top quark of the order of $2 \mathrm{TeV}$ or larger [51-55]. Disregarding sleptons for the moment, the remaining states of the MSSM then are the superpartners of the weak gauge bosons and Higgs doublets, and additional Higgs bosons. Assuming this low-energy spectrum leads to a particularly simple set of states, whose dynamics are determined by only a few parameters. The neutralinos appear as mixtures of the Bino, Wino, and the Higgsinos whose mass matrix is given by

$$
M_{N}=\left(\begin{array}{cccc}
M_{1} & 0 & -c_{\beta} s_{W} m_{Z} & s_{\beta} s_{W} m_{Z} \\
0 & M_{2} & c_{\beta} c_{W} m_{Z} & -s_{\beta} c_{W} m_{Z} \\
-c_{\beta} s_{W} m_{Z} & c_{\beta} c_{W} m_{Z} & 0 & -\mu \\
s_{\beta} s_{W} m_{Z} & -s_{\beta} c_{W} m_{Z} & -\mu & 0
\end{array}\right),
$$

where $c_{W}=\cos \theta_{W}, s_{W}=\sin \theta_{W}$, and $\theta_{W}$ is the weak-mixing angle, $c_{\beta}=\cos \beta, s_{\beta}=\sin \beta$, and $\tan \beta=\left\langle H_{u}^{0}\right\rangle /\left\langle H_{d}^{0}\right\rangle$ is the ratio of the vacuum expectation values of the Higgs doublets. The mass $M_{1}$ is the Bino mass parameter, $M_{2}$ and $\mu$ are the Wino and Higgsino mass parameters, respectively. Similarly, the chargino states appear as mixtures of charged Wino and Higgsino components with mass matrix

$$
M_{C}=\left(\begin{array}{cc}
M_{2} & \sqrt{2} s_{\beta} m_{W} \\
\sqrt{2} c_{\beta} m_{W} & \mu
\end{array}\right) .
$$

For a more detailed review of the resulting mass eigenstates and couplings see ref. [18] and references therein. Including the Higgs sector of the MSSM, the low energy theory results in a rich phenomenology where the relevant parameters are $\left\{M_{1}, M_{2}, \mu, \tan \beta\right\}$ and the masses of the heavy Higgs bosons, which are characterized with the CP-odd Higgs mass parameter $m_{A}$. In the case when the Wino is also decoupled the lightest electroweakinos are Bino-, and Higgsino-like. In this limit, the results we present in the following sections relies mainly on only four parameters $\left\{M_{1}, \mu, \tan \beta, M_{A}\right\}$.

\subsection{Direct electroweakino production}

The direct production of electroweakino states is governed by the interchange of $W^{ \pm}$and $Z$ gauge bosons (see for instance, ref. [18]). Since the Bino does not couple to the gauge bosons, all production modes are controlled by the Higgsino couplings to gauge bosons, and the production of a particular mass eigenstate will depend on its component of the Higgsino 
states. Assuming the lightest supersymmetric particle is mostly a Bino, its production, although kinematically favored, may only happen through its mixing with the neutral Higgsino states. Pair production of Bino states may be measured via its recoil against jets, in the jets plus missing energy channel. However, the production cross section tends to be too small to be competitive with other search channels. Binos may be hence mostly produced in association with the charged and neutral Higgsino states, leading after the decay of the heavier states to final states consisting of $h, Z$ or $W^{ \pm}$plus missing energy. However, unless the Bino and Higgsino masses are close to each other, the cross sections are again too small to make these competitive search channels.

Much more promising is the search for electroweakinos via the production of the heavier, but mainly Higgsino states. Since it is not hampered by mixing, this search is only limited by the kinematic effects related to low production cross sections associated with high mass states. Also, in the limit of heavy superpartners, the Higgsino cross sections are smaller than the Wino cross sections, implying also a smaller reach than in the case of light Winos. Interestingly enough, since the scalar leptons couple to the Higgsino states in a way proportional to Yukawa couplings, the search for Higgsinos tends to be minimally affected by the presence of such states, which in this scenario will therefore mainly decay into leptons and missing energy and can be searched separately from the Higgsino states. This is relevant, since light sleptons may contribute to an explanation of the $g-2$ anomaly [56-61] . Observe, however, that the contributions to the muon anomalous magnetic moment are highly reduced in the case of heavy Winos. In fact, even if the slepton masses were only a few hundreds of $\mathrm{GeV}$, the contributions to $(g-2)_{\mu}$ in the region of parameters we are considering, for moderate values of $\mu$ and $\tan \beta$, is only a few times $10^{-10}$ and not large enough to fit the Brookhaven experiment observation [62],

$$
\delta a_{\mu}^{\exp } \simeq(27 \pm 7 \pm 5) \times 10^{-10}
$$

where the errors are associated with experimental and theoretical uncertainties. Fitting the Brookhaven experiments would demand large values of $\tan \beta$ pushing the Higgs masses to values larger than $1 \mathrm{TeV}[63,64]$, for which the Higgs boson decay into electroweakinos ceases to be a competitive search channel.

Regarding the Higgsino being a Dark Matter candidate, if one assumes that the Dark Matter relic density comes from thermal annihilation, one needs to mix Higgsino and Bino appropriately to reach the right relic abundance. This is the so-called well tempered neutralino region [65], which tends to be in tension with direct detection experiments [6671]. However, the relic density constraints can be easily evaded in the case of light Binos by the assumption of late entropy production (see, for instance, ref. [72]). Other ways to avoid the relic density constraints in the region of parameters we are concentrating on include coannihilation, $Z$-resonance annihilation , $h$-resonance annihilation (see, for instance, refs. [73-76] ). Moreover, it is easy to weaken the spin-independent direct Dark Matter search constraints by simply concentrating on negative values of $\mu$ (see refs. [71, 7783]). Though, it is worth noting that the constraints from spin-dependent Dark Matter searches will still apply [84, 85]. 

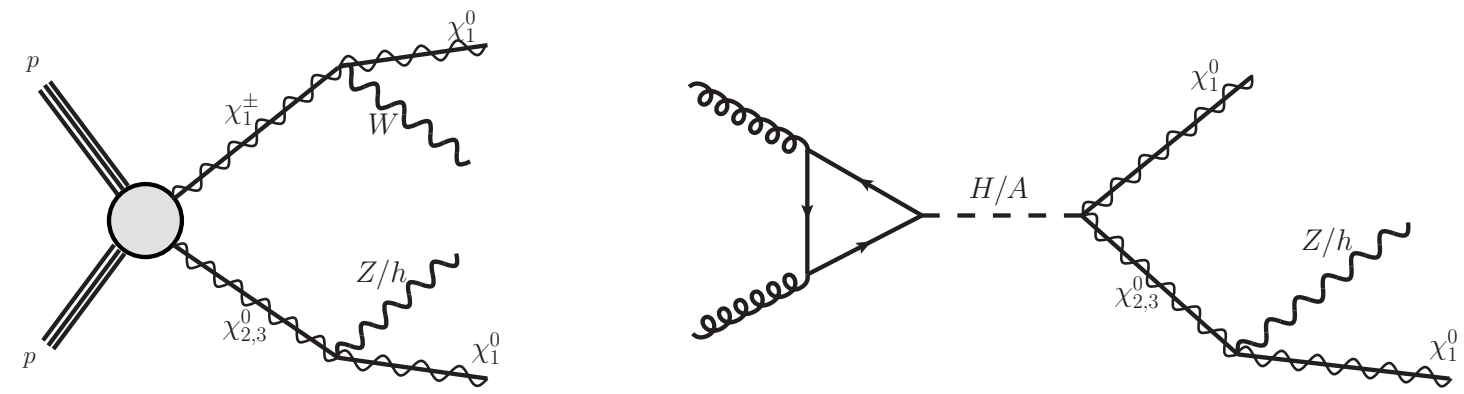

Figure 1. Representative decay topologies of electroweakino production at the LHC. Left: direct production channels resulting from pair producing a chargino and neutralino. Right: the Higgs portal channel resulting from the production of a heavy neutral Higgs boson decaying to neutralino pairs.

For the reasons discussed above, we will be mostly interested in the production of mainly Higgsino states with negative values of $\mu$. The spectrum will consist of two neutral Majorana states, the second and third lightest neutralinos, and one charged Dirac state. The associated direct production of the charged and neutral states, mediated mostly by the charged gauge boson $W^{ \pm}$carries the largest cross section and it is represented in figure 1. Since the second and third lightest neutralinos are close in mass, provided it is kinematically allowed, they will mostly decay into either $Z$ or light Higgs $h$ final states plus missing energy. The chargino state, instead will decay into $W^{ \pm}$and missing energy

$$
p p \rightarrow \chi_{1}^{ \pm}+\chi_{h}^{0} \rightarrow W^{ \pm}+Z / h+\mathbb{E}_{T},
$$

where $h=2,3$. We will concentrate in the region of parameters where the second and third lightest neutralino can decay into on-shell $Z$ gauge bosons. Therefore, considering the decay of the $W^{ \pm}$into lepton states, the most interesting final states will be either trileptons plus missing energy or pairs of bottom quarks plus one lepton plus missing energy. Considering the decay of the $W^{ \pm}$into hadrons, the most interesting channel is two leptons (from $Z$ ) or two bottoms (from $h$ ) and missing energy.

In figure 2 we present the dependence of the associated production cross section as a function of the Higgsino mass parameter $\mu$ that we take to be negative for this consideration, and that represents the overall scale of the Higgsino masses (there is a small dependence of the Higgsino spectrum on the sign of $\mu$, which is however, only significant for low values of $\mu$, of the order of $100 \mathrm{GeV}$ ). We also represent, for comparison, the production of Winos, as a function of the Wino mass parameter $M_{2}$, making evident the larger cross sections associated with these states.

The branching ratios also play an important role. The presence of two different Higgsino states imply that their decay branching ratios will not be equal. These two Higgsino states are close in mass, particularly for masses larger than Higgs mass, $125 \mathrm{GeV}$, for which also the dependence on the sign of $\mu$ becomes less significant. It is therefore useful to represent the sum of the branching ratios of the decay of the second and third lightest neutralinos into $Z$ and Higgs final states. These are given in figure 3. In each case, we 


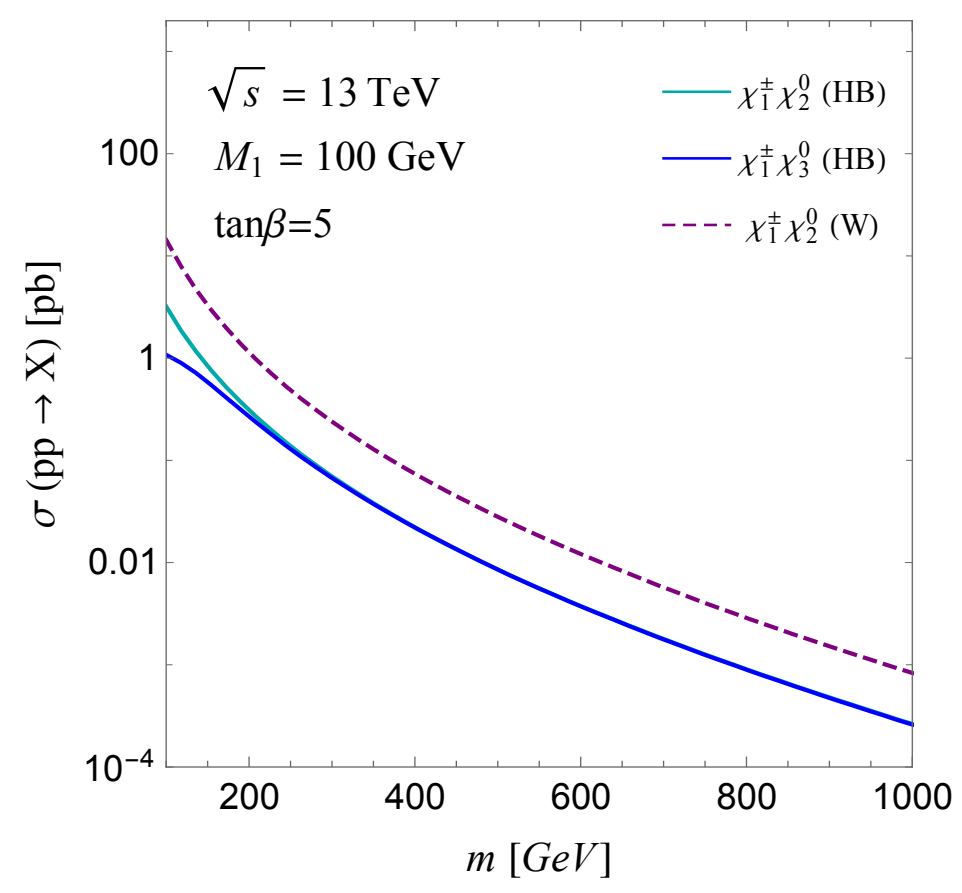

Figure 2. Comparison of the LO direct production cross sections of electroweakinos in the pure Wino scenario (dashed lines) and the Bino-Higgsino scenario (solid lines) with $M_{1}=100 \mathrm{GeV}$ and $\tan \beta=5$, assuming that scalar superpartners are decoupled. In the former case, $\mu=-2 \mathrm{TeV}$ and $m=M_{2}$ is varied. In the latter, $M_{2}=2 \mathrm{TeV}$ and $m=|\mu|$ is varied.

calculate the branching ratio using the code Spheno $[86,87]$ as functions of the parameters

$$
M_{1} \in[5,500], \quad-\mu \in[100,900],
$$

assuming $M_{2}=2 \mathrm{TeV}, \tan \beta=5$, and $M_{A}=1 \mathrm{TeV} \cdot{ }^{1}$ For large values of $|\mu|$ the two neutralino Majorana states behave effectively like a single neutral Dirac state and, due to the Goldstone equivalent theorem, one expects that, approximately, the neutral states will decay 50 percent of the time into $Z$ and 50 percent of the time into $h$ final states, something that is evident from figure 3 .

In the low mass range, provided both neutralinos become lighter than the Standard Model-like Higgs boson, they will decay into $Z$ final states 100 percent of the time. However, we remark that for low values of $\tan \beta$ the splitting can be large enough so that whenever one neutralino is lighter than the Higgs the other becomes heavier, up to the point when the lightest neutralino can no longer decay into on-shell $Z$ bosons. Apart from this, the total branching ratios of electroweakinos to the $Z$ or Higgs boson display the same behavior for values of $\tan \beta$ that we consider.

\footnotetext{
${ }^{1}$ Note that for lower values of the heavy Higgs mass, such that $M_{A}<m_{\chi_{1}^{ \pm}}+m_{\chi_{1}^{0}}$, decay channels of electroweakinos to heavy Higgs bosons may become kinematically open. However, the corresponding branching ratios of these channels would be two small to be of any relevance.
} 

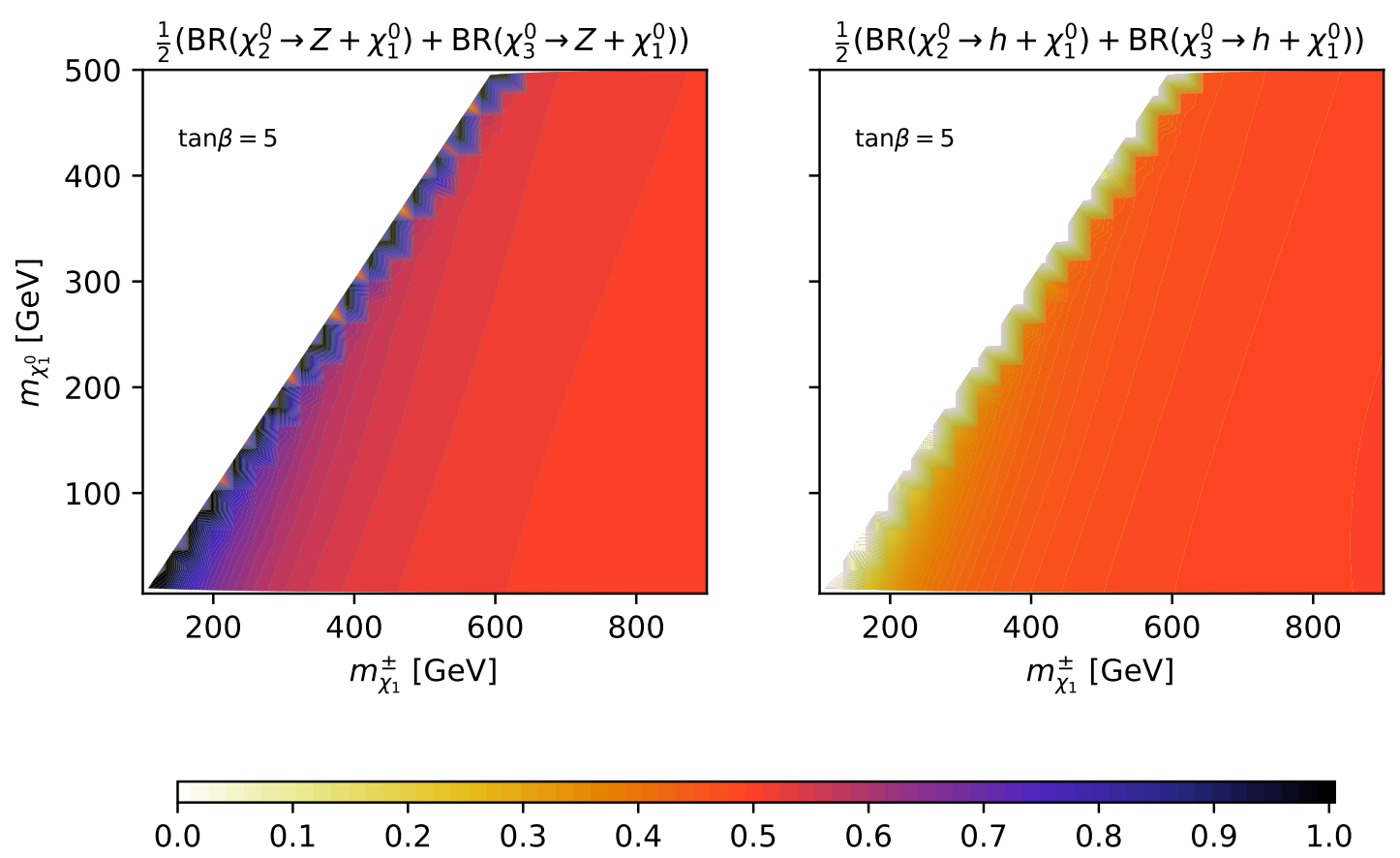

Figure 3. Left: sum of the total branching ratio of Higgsinos to the $\mathrm{Z}$ boson, $\chi_{2,3}^{0} \rightarrow Z+\chi_{1}^{0}$, normalized by $1 / 2$. Right: sum of the total branching ratio of Higgsinos to the SM Higgs, $\chi_{2,3}^{0} \rightarrow$ $h+\chi_{1}^{0}$, normalized by $1 / 2$. We show contours resulting from the scan in eq. $(2.5)$, for $\tan \beta=5$.

\subsection{Electroweakinos from heavy Higgs production}

The production of electroweakinos from the decay of heavy Higgs bosons has a different parametric dependence than the direct production. This is due to the fact that, contrary to the coupling to $Z$ gauge bosons, pure Higgsino states do not couple to the heavy Higgs boson due to gauge invariance, and the coupling always involves mixed Higgsino and gaugino (Bino) components. Hence, the heavy Higgs decays into a mostly Higgsino and a mostly Bino state is preferred with respect to both the relevant couplings and kinematics.

There are hence two main possibilities. Either the lightest neutralino is produced from the decay of neutral Higgs bosons, in association with the second and third lightest neutralinos, or it is produced in association with the chargino states, coming from the decay of the charged Higgs boson. In the case of neutral Higgs bosons, which is diagrammatically represented in figure 1, where the second and third lightest neutralino can decay into either a $Z$ or a Higgs boson $h$ (the heavier Higgs bosons are highly degenerate in mass), leading to interesting mono-Higgs and mono- $Z$ final states. Among the mono- $Z$ final states, the decay into two leptons becomes particularly interesting as has already been emphasized in refs. [39]-[50]. 


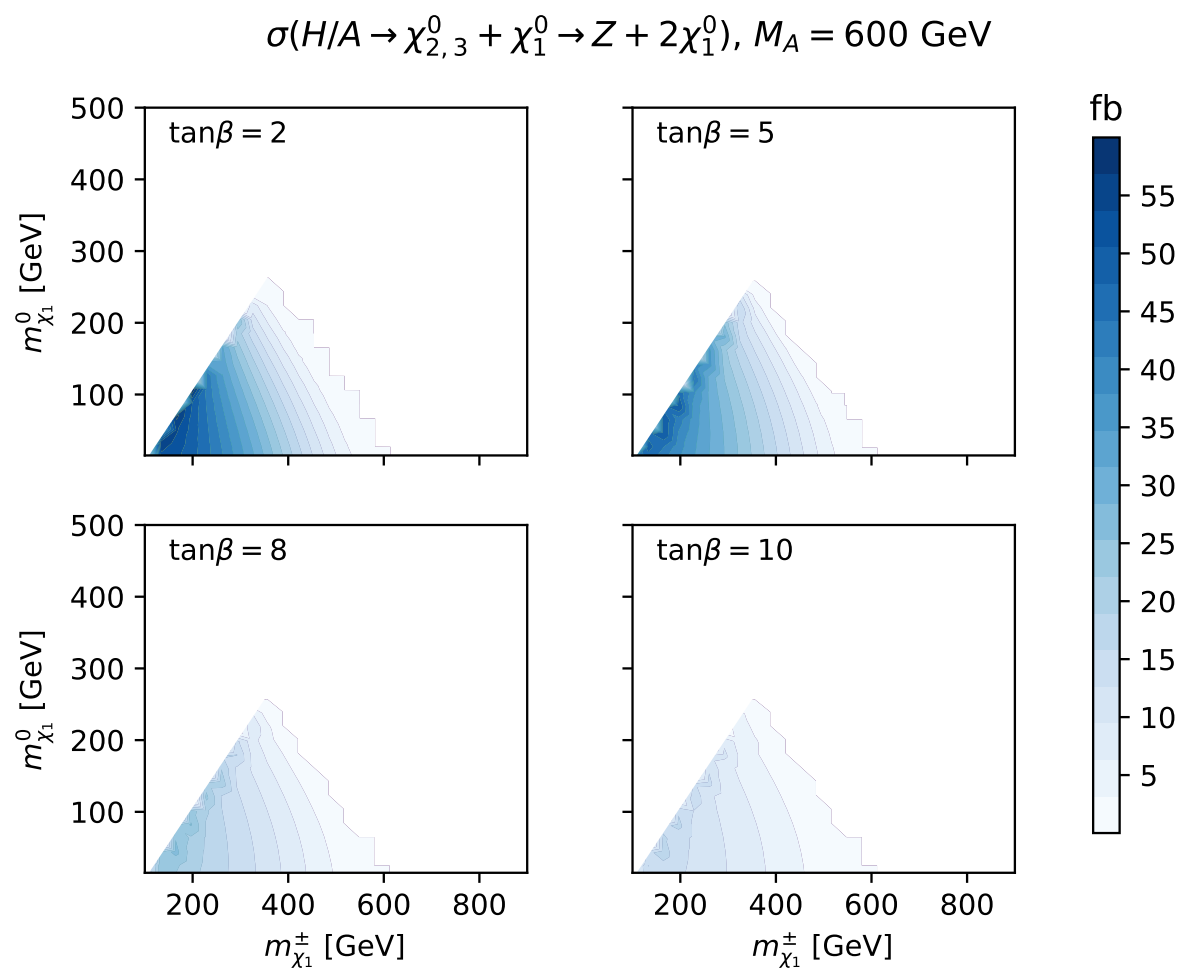

Figure 4. The production cross-section for the process $H / A \rightarrow \chi_{2,3}^{0} \chi_{1}^{0} \rightarrow Z+2 \chi_{1}^{0}$ for higgsino-like electroweakinos, with heavy scalar mass $m_{A}=600 \mathrm{GeV}$ and $\tan \beta=2,5,8,10$ respectively.

The charged Higgs production mode is also interesting. Charged Higgs, however, can only be produced at sufficiently high rates in association with top and bottom quarks, implying significantly smaller cross sections than the ones associated with neutral gauge bosons. Also, the final state is similar to the top-quark pair production mode, albeit with significant missing energy. Hence the reach is hampered by the large $\bar{t} t$ background. For these reasons, in this work we shall not concentrate on this mode, but we plan to come back to it in an independent analysis.

\section{Reach of direct production searches of higgsino states at higher lumi- nosities}

In order to compute the reach for Higgsino states in the direct production mode, we have considered a number of recent ATLAS studies, refs. [7, 9, 10, 12], in which they present bounds on the masses of charginos and neutralinos at the $13 \mathrm{TeV}$ LHC for luminosities ranging from $36 \mathrm{fb}^{-1}$ to $139 \mathrm{fb}^{-1} .^{2}$ The bounds presented in these studies assume that the electroweakino spectrum results from parameters aligned with the pure Wino scenarios and that the corresponding branching ratios of neutralinos into either the $Z$ boson or SM $h$ are 100 percent, depending on the study. We have recasted each bound including the cross sections associated with the production of Higgsino-like states, and incorporated the branching

\footnotetext{
${ }^{2}$ The CMS study shows similar sensitivities when luminosity is the same, see [5].
} 
ratios of the neutralino states for a given chargino mass (which is also degenerate in mass with the neutral Higgsino states for large values of $\mu$ ) resulting from the scan of eq. (2.5).

To obtain the relevant cross section starting from the parameters $M_{1}, \mu$ and $\tan \beta$, mass eigenstates, mixings, and branching ratios are calculated with FeynHiggs [55, 88-94] linked with SPheno. The branching ratios of EW-inos to SM gauge bosons have also been checked with SUSY-HIT [95]. When presenting bounds on direct search channels we consider fixed $\tan \beta=5$. In the Higgsino-Bino limit, the $\tan \beta$ dependence on the relevant branching ratios follows as $\operatorname{BR}\left(\chi_{2}^{0} \rightarrow Z / h+\chi_{1}^{0}\right) \sim \frac{1}{2}(1 \mp \sin (2 \beta))$ and $\operatorname{BR}\left(\chi_{3}^{0} \rightarrow Z / h+\chi_{1}^{0}\right) \sim \frac{1}{2}(1 \pm \sin (2 \beta))$, when $|\mu|-M_{1}$ is significantly larger than $m_{h}$ [96]. Thus, since we sum both contributions from $\chi_{2}^{0}$ and $\chi_{3}^{0}$ in the production channels, the overall dependence of the total cross is dominated by kinematics rather than the coupling, as expected by the Goldstone Boson Equivalence theorem. In contrast, the Higgs production channel will depend strongly on $\tan \beta$. We will explore this complementarity of the parameter space further in the following section.

LO cross sections were generated and cross checked between MadGraph5 [97] and Prospino2.1 [98]. For chargino masses $\sim 100 \mathrm{GeV}$ the K-factors between LO and NLO elecroweakino cross sections are about $K \simeq 1.3$ [99]. We find that the K-factors decrease to $K \simeq 1.1$, once the mass of the chargino reaches $\sim 500 \mathrm{GeV}$. As this variation occurs in a region which is relevant to the current bounds, we have used the NLO cross sections from Prospino2.1 in our results. For each chargino-neutralino pair in the scan we then compare the cross section to the corresponding $95 \%$ CL upper limit, $\sigma_{\text {limit }}^{\text {up }}$, taken from HEP-Data

$$
N_{\text {sig }}\left(N_{\text {bkg }}, 95 \%\right)=\sigma_{\text {limit }}^{\text {up }} \epsilon_{\text {eff }} L .
$$

Assuming that the background efficiencies remain the same, we then find the bound for Higgsino-Bino masses via

$$
\sigma\left(p p \rightarrow \chi_{1}^{ \pm} \chi_{2,3}^{0}\right) \mathrm{BR}\left(\chi_{1}^{ \pm} \rightarrow W^{ \pm}+\chi_{1}^{0}\right) \mathrm{BR}\left(\chi_{2,3}^{0} \rightarrow Z / h+\chi_{1}^{0}\right)=\sigma_{\text {limit }}^{\text {up }} .
$$

Finally, we extrapolate the existing bounds for the $13 \mathrm{TeV}$ LHC to luminosities of $300 \mathrm{fb}^{-1}$ and $3 \mathrm{ab}^{-1}$

$$
\sigma_{\text {limit }}^{\text {up; } \mathrm{L}^{\prime}}=\sqrt{\frac{L^{e x p}}{L^{\prime}}} \sigma_{\text {limit }}^{\text {up }},
$$

where $L^{\exp }$ is the corresponding luminosity for each analysis that we have considered.

Inspection of the LHC experiment analyses reveal that the most powerful searches are associated with the tri-lepton final states and the Higgs final state. In the following subsection, we review the status of the different search channels for Higgsino-like states. We note that since the efficiencies tend to be improved with further analyses and the energy will be eventually upgraded to values of order of $14 \mathrm{TeV}$ our analysis should be considered as a conservative one.

\subsection{ZW channels}

This is the traditional search channel for electroweakinos and leads to very powerful results at high luminosities. The ATLAS collaboration has explored this channel using a variety of techniques and final states. As mentioned, final states with three leptons [12] or two 

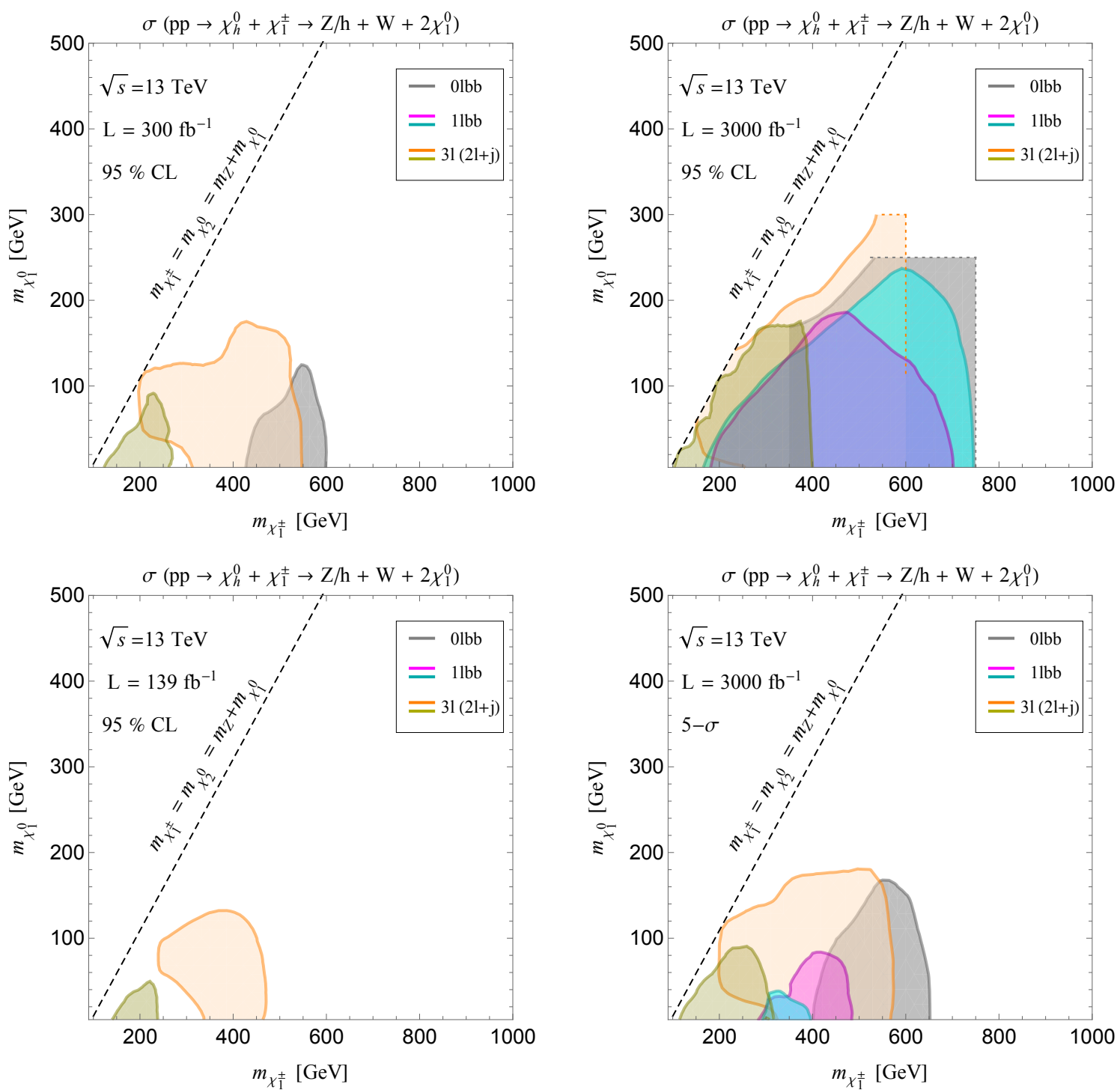

Figure 5. Constraints on the Bino-Higgsino scenario projected to 300 and $3000 \mathrm{fb}^{-1}$ at $95 \%$ confidence level in the top panel, $139 \mathrm{fb}^{-1}$ at $95 \%$ confidence level and $3000 \mathrm{fb}^{-1}$ at $5 \sigma$ in the lower panel. We choose $\tan \beta=5$, but the results depend very weakly on this choice for the range

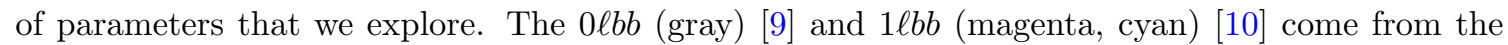
$\chi_{2,3}^{0} \chi_{1}^{ \pm} \rightarrow h W+2 \chi_{1}^{0}$ channel, with $h \rightarrow \bar{b} b$ and $W$ decay to hadronic or leptonic final states. The $3 \ell$ (dark yellow) [12] and $3 \ell / 2 \ell+\mathrm{j}$ (orange) [8] come from the $\chi_{2,3}^{0} \chi_{1}^{ \pm} \rightarrow Z W+2 \chi_{1}^{0}$ channel, with $Z \rightarrow 2 \ell$. For the gray and orange shaded region, there are dotted lines cutting off the high mass region because $\sigma_{\text {limit }}$ is not provided from HEP-Data. 
leptons with jets [7] provide the strongest reach. After recasting the LHC analyses for the case of Higgsinos and using both the Recursive Jigsaw reconstruction method as well as the standard ones, we observe the sensitivity for Higgsino-like electroweakinos at a luminosity of $300 \mathrm{fb}^{-1}$ in the top left panel of figure 5 . The LHC should be capable of reaching Higgsino masses of about $500 \mathrm{GeV}$, but only for lightest neutralino masses that are lower than $200 \mathrm{GeV}$, and far from the kinematic threshold for the decay of the second and third lightest neutralino into $Z$ states. This is due to the fact that, for masses close to this kinematic threshold, the leptons carry low $p_{T}$ values. This is one of the main advantages of the Higgs production mode, where the leptons will receive higher $p_{T}$ contributions due to the high $p_{T}$ of the electroweakinos proceeding from the decay of the heavy Higgs bosons.

In the top right panel of figure 5 , at higher luminosity of $3000 \mathrm{fb}^{-1}$, the situation is highly improved. On one hand, Higgsino masses up to $750 \mathrm{GeV}$ could be reached in this case, for lightest neutralino masses up to about $300 \mathrm{GeV}$. The reach for $3 \ell$ (dark yellow line) stops at the dotted lines. In this case, $\sigma_{\text {limit }}^{\text {up }}$ is not provided for the higher mass region in the current searches. However, it is expected that updated searches would be sensitive to this region. In general, this is a highly interesting reach, that covers the region of Higgsino masses one would associate with a natural low energy supersymmetry scenario. But the kinematic limitations close to threshold are still present, although only for higher lightest neutralino masses.

\section{2 hW channels}

A secondary channel to probe the production of charginos and neutralinos occurs when the neutralinos decay to a SM Higgs boson. The ATLAS collaboration has studied this channel for electroweakino pair production of $\chi_{1}^{ \pm} \chi_{2}^{0}$. Similarly, the production cross sections assumed couplings associated to Wino-like couplings and the decay branching ratio of $\chi_{1}^{ \pm} \rightarrow W^{ \pm} \chi_{1}^{0}$ and $\chi_{2}^{0} \rightarrow h \chi_{1}^{0}$ are both $100 \%[9,10]$. The final states considered in these studies are $0 \ell+\bar{b} b, 1 \ell+\bar{b} b, 1 \ell+\gamma \gamma$ and $\ell^{ \pm} \ell^{ \pm}$, which arise from a combination of leptonic and hadronic decays of the $W^{ \pm}$, and SM Higgs decay into $\bar{b} b, \gamma \gamma$ and $2 \ell$. From their results we find that the $0 \ell+\bar{b} b$ and $1 \ell+\bar{b} b$ channels provide the strongest bound, therefore to explore the ultimate reach for Higgsino-like electroweakinos we only recast these channels.

We find that these channels provide a 95\% CL reach for larger chargino masses almost up to 600 (750) GeV for luminosity of $300(3000) \mathrm{fb}^{-1}$, as shown in the top panel of figure 5 . For luminosity of $300 \mathrm{fb}^{-1}$, only the $0 \ell+\bar{b} b$ channel provides constraints, for $m_{\chi_{1}^{ \pm}}$between $400-600 \mathrm{GeV}$ and very light $\chi_{1}^{0}$, while the bound from the $1 \ell+\bar{b} b$ channel vanishes. This already shows the need for higher luminosities to search for higgsino-like electroweakinos in this decay channel. For luminosity of $3000 \mathrm{fb}^{-1}$, we see a dramatic enhancement of the constrained region for both $0 \ell+\bar{b} b$ and $1 \ell+\bar{b} b$ channels.

Moreover, in the bottom right panel of figure 5 we show the $5 \sigma$ reach for direct production of Higgsino-like states at HL-LHC both for the $Z W$ and $h W$ channels. Comparing with the bottom left panel of figure 5 , we see that it is still possible to discover light electroweakinos in the HL-LHC era in regions of parameters not already probed at current collected luminosity of $139 \mathrm{fb}^{-1}$. Our results are consistent with other projections to future upgrades at the LHC $[96,100,101] .^{3}$

\footnotetext{
${ }^{3}$ For other theoretical studies in this direction see [74].
} 


\section{Reach of the Higgs decay production mode at higher luminosities}

Although there are already a few theoretical analyses of this mode [39]-[50], there is to our knowledge no dedicated search for electroweakinos in this production mode. Therefore we performed our own analysis of the background and production rates. ${ }^{4}$ We concentrated only on the gluon fusion mode, and on the lepton decays of the $Z$ bosons proceeding from the decay of the second and lightest neutralinos in the lepton plus missing energy analysis. Thus, the relevant signal results in two opposite-sign, same flavor leptons with significant missing energy

$$
g g \rightarrow H / A \rightarrow \chi_{h}^{0}+\chi_{1}^{0} \rightarrow \ell^{+} \ell^{-}+\mathbb{E}_{T},
$$

where we sum the channels from heavy neutral scalar $H$ and pseudoscalar $A$ production, and $h=2,3$.

The production of Higgs bosons become significant at either small or large values of $\tan \beta$, for which the gluon fusion cross section is proportional to the large top and bottomquark Yukawa couplings, respectively. The main couplings governing the production cross section are given by

$$
g_{H t \bar{t}} \simeq g_{A t \bar{t}} \simeq \frac{m_{t}}{v \tan \beta}
$$

and

$$
g_{H b \bar{b}} \simeq g_{A b \bar{b}} \simeq \frac{m_{b} \tan \beta}{v\left(1+\Delta_{b}\right)},
$$

where $m_{t}, m_{b}$ and $v$ are the running top-quark and bottom-quark masses and the Higgs vacuum expectation value $v \simeq 174 \mathrm{GeV}$, respectively. The factor $\Delta_{b}$ is associated with a threshold correction [102, 103], induced by the decoupling of the supersymmetric particles [104-106], which tends to be suppressed for low values of the Higgsino mass parameter compared to the heavy colored particles. Moreover, $\Delta_{b}$ becomes significant in this case only for very large values of $\tan \beta$, for which the heavy Higgs bosons are heavily constrained via their decay into $\tau$ leptons $[63,64]$.

Regarding branching ratios, it is important to remark that the same couplings that control the production cross sections also control the branching ratios into top and bottom quarks. Hence, assuming Higgs masses above the top quark pair production threshold, the gain in production cross section at low and large values of $\tan \beta$ is compensated by the reduction of the branching ratio of the decay into electroweakinos. Overall, considering both the Higgs production and decay branching ratios while concentrating on the gluon fusion analysis, we find that the total production rate coming from the decay into electroweakinos is enhanced for lower values of $\tan \beta$ and decreases slowly for larger values of $\tan \beta$.

In the MSSM, for moderate values of the Higgsino mass parameter $\mu$, low values of $m_{A}$ lead to a modification of the Higgs couplings which therefore affect the agreement between the theoretical predictions and the Higgs precision measurements [107, 108]. The modifications of the Higgs couplings are approximately independent of $\tan \beta$ and lead to a bound of about $600 \mathrm{GeV}$ on $m_{A}$ [38]. Observe, however, that these bounds may be modified

\footnotetext{
${ }^{4}$ We have closely followed the analysis strategy given in ref. [39], where the same signature has been studied exclusively in the compressed region, $m_{\chi_{2,3}^{0}}-m_{\chi_{1}^{0}} \simeq m_{Z}$ and we find a similar reach.
} 
considering the low energy theory to proceed from, for instance, the NMSSM in the limit of heavy singlet [109], that may arise dynamically from the renormalization group evolution at low energies $[110,111]$. In this article, we have mainly focused on the region of masses higher than $600 \mathrm{GeV}$, since they allow to avoid the precision measurement constraints in the MSSM, but also enhance the reach for heavier electroweakinos.

Beyond the precision measurement constraints, other relevant constraints come from the search for heavy Higgs bosons decaying into SM final states. As discussed before, in the MSSM, the decays of the heavy Higgs to bottom quarks and tau leptons are enhanced by large $\tan \beta$. The $H / A \rightarrow \tau^{+} \tau^{-}$decay channel has been studied in the ATLAS Run-II search $\left(139 \mathrm{fb}^{-1}\right)[64]$ in the case where at least one tau lepton decays hadronically and assuming that all superpartners are heavy enough that the $H / A$ Higgs bosons can not decay into them. In this case, the current bounds would exclude values of $\tan \beta \gtrsim 8$ for $m_{A}=600 \mathrm{GeV}$. However, in the present case the heavy neutral Higgses can decay into a Higgsino-Bino pair with branching ratios close to $30 \%$ which, for given $m_{A}$, alleviates the upper limit on $\tan \beta$.

Recently, the comparison of the current and projected bounds between these two scenarios has been studied in [112]. They find that for scenarios with light electroweakinos the bound from $H / A \rightarrow \tau^{+} \tau^{-}$excludes $\tan \beta \gtrsim 15$, for $m_{A}=600 \mathrm{GeV}$ and luminosity of $36 \mathrm{fb}^{-1}$. While for the HL-LHC at $14 \mathrm{TeV}$ and luminosity of $3 \mathrm{ab}^{-1}$, they project this bound to be $\tan \beta \gtrsim 8$. Additionally, constraints for the $H / A \rightarrow \bar{b} b$ decay have been presented in [113]. However, the bound is quite weak and only excludes values of $\tan \beta>20$, for the same region of heavy Higgs masses. As we shall see, irrespectively of these bounds, the sensitivity of the search for electroweakinos proceeding from the decay of heavy Higgs bosons is highly reduced for values of $\tan \beta>10$, even for low masses of the heavy Higgs.

For lower $\tan \beta$, there exist constraints from the decays of the charged Higgs bosons, $H^{+} \rightarrow t \bar{b}$, and also the neutral Higgs channel, $H / A \rightarrow \bar{t} t$. In the case of the charged Higgs the bounds become weak in the region of parameters we are considering, as can be seen from the results of ref. [114]. For instance, for $\tan \beta=0.5$ the mass of the charged Higgs should satisfy $m_{H^{ \pm}}>1.2 \mathrm{TeV}$, while for $\tan \beta=1$ there are no constraints for $m_{H^{ \pm}}$. For the decay of the neutral Higgs bosons to $\bar{t} t$, the projected HL-LHC sensitivity is $\tan \beta \lesssim 2$ for a heavy Higgs mass $m_{A}=600 \mathrm{GeV}$ [103]. However, considering top associated production can mildly increase this sensitivity to $\tan \beta \lesssim 3$. The current limits for the $\bar{t} t$ channel for $m_{A}=600 \mathrm{GeV}$ is about $\tan \beta \lesssim 1.5$, coming from the analysis of the CMS $13 \mathrm{TeV} 36 \mathrm{fb}^{-1}$ data [115]. In our analysis, we shall limit our analysis to values of $\tan \beta \geq 2$. Also, unless the stop masses are larger than $10^{5} \mathrm{GeV}$, it is impossible to obtain the proper Higgs mass in the MSSM for values of $\tan \beta$ lower than 2 [51-55].

Another possible search channel for the heavy Higgs follows from the same production mechanism but followed by the heavier neutralinos decaying instead to the SM Higgs boson, $\chi_{2,3}^{0} \rightarrow h+\chi_{1}^{0}$, followed by the Higgs decay to bottom quarks. Despite the fact that this decay chain can have a sizable rate, depending on $m_{A}$ and $\tan \beta$, this signal suffers from multiple QCD backgrounds. We have estimated the signal significance of this channel using the background and analysis of ref. [116]. We find that the reach of this channel will not be competitive in comparison to direct searches or the dilepton Higgs channel. Thus, we will not explore this possibility in this work. 

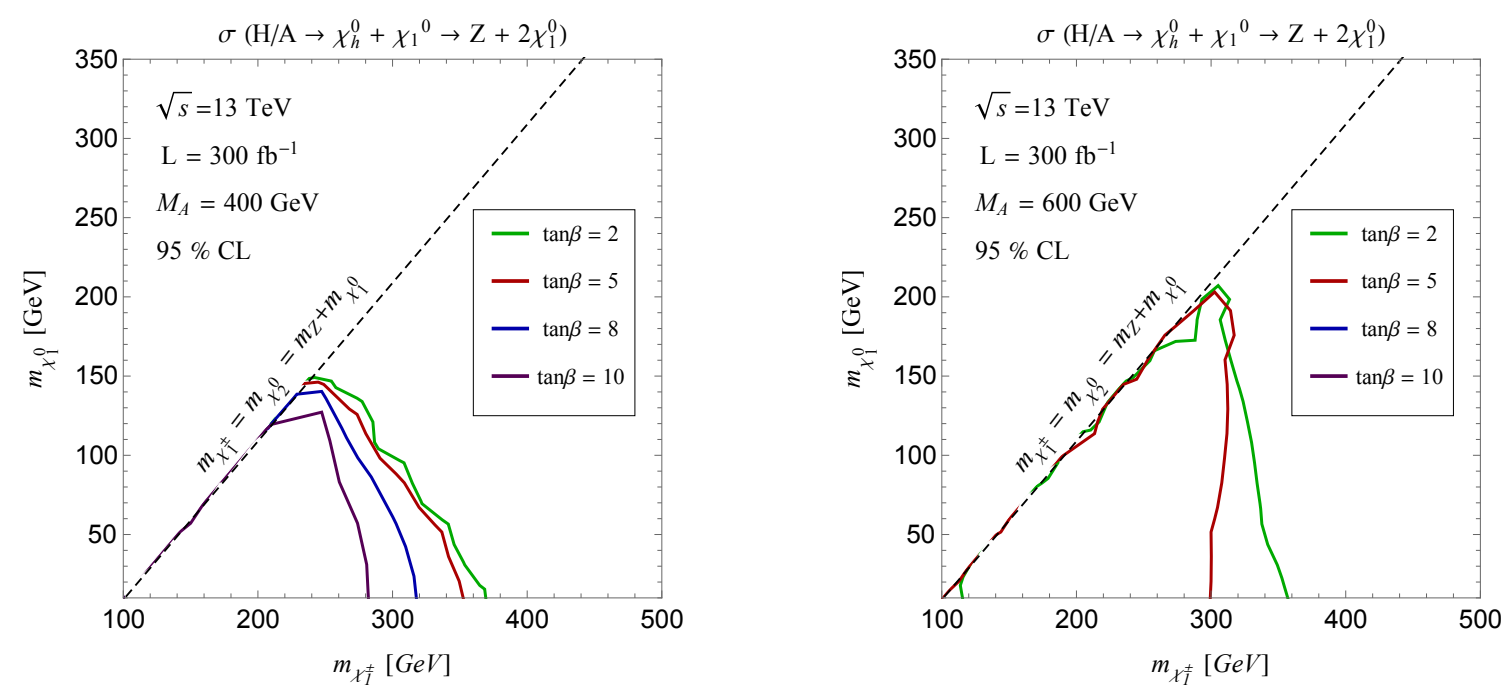

Figure 6. $95 \%$ CL bounds on the heavy neutral Higgs decay into electroweakinos $H / A \rightarrow \chi_{h}^{0} \chi_{1}^{0} \rightarrow$ $Z+2 \chi_{1}^{0}$, with $Z \rightarrow \ell^{+} \ell^{-}$. With integrated luminosity of $300 \mathrm{fb}^{-1}$ and $m_{H / A}=400 \& 600 \mathrm{GeV}$, the sensitivities for electroweakinos are shown as contours in the $m_{\chi_{1}^{ \pm}} m_{\chi_{1}^{0}}$ plane for $\tan \beta=$ $2,5,8, \& 10$. For $m_{H / A}=600 \mathrm{GeV}$ the bounds vanish for values of $\tan \beta \gtrsim 5$.

To compute the resulting bounds, requiring that events contain a significant amount of missing energy drastically reduces the contribution from QCD backgrounds. The relevant backgrounds then come from di-boson production, $p p \rightarrow V V$, with $V=W, Z$. Further requiring that one pair of opposite-sign, same flavor leptons reconstruct the $Z$-boson mass, $85 \mathrm{GeV}<m_{\ell \ell}<95 \mathrm{GeV}$, leaves the $Z Z$ channel as the most relevant source of background. Apart from large $\mathbb{E}_{T}$ in the events, the signal significance can be further improved by using the modified clustered transverse mass introduced in ref. [39],

$$
m_{c T}^{2}\left(\ell \ell, \mathbb{E}_{T}\right)=2 \times\left(\left(\left|\vec{p}_{T}^{\ell \ell}\right|+\left|\vec{p}_{T}\right|\right)^{2}-\left|\vec{p}_{T}^{\ell \ell}+\vec{p}_{T}\right|^{2}\right),
$$

where $\vec{p}_{T}^{\ell \ell}$ is the transverse momentum of the di-lepton system, and $\vec{p}_{T}$ is the two-vector of the missing transverse energy. For more details of the kinematics and impact of the cuts see ref. [39]. We generate background and signal using MadGraph5 followed by shower and detector simulation with Pythia8 $[117,118]$ and Delphes [119] respectively. For a given heavy Higgs mass and $\tan \beta$, we calculate the signal significance in the chargino-neutralino plane, calculated from eq. (2.5), by optimizing lower and upper cuts on $\mathbb{E}_{T}$ and $m_{c T}^{2}$, respectively.

Our results for $300 \mathrm{fb}^{-1}$ are presented in figure 6 in the $m_{\chi_{1}^{ \pm}}-m_{\chi_{1}^{0}}$ plane. Viewing the bounds in this way allows for a straightforward comparison with the direct production search channels in the same parameter space. For $300 \mathrm{fb}^{-1}$, the LHC sensitivity for heavy Higgs bosons decaying into electroweakinos is limited to relatively low values of $m_{A}$, that as argued before would lead to tension with Higgs precision measurements, unless one goes beyond the pure MSSM description. In the left panel, we show the bounds for $m_{A}=400 \mathrm{GeV}$. For $\tan \beta=2$, the sensitivity covers almost the entire region that is kinematically allowed. Whereas for $\tan \beta=10$, the sensitivity reaches to chargino masses of almost $300 \mathrm{GeV}$, and slightly above $100 \mathrm{GeV}$ for the lightest neutralino mass. In the 

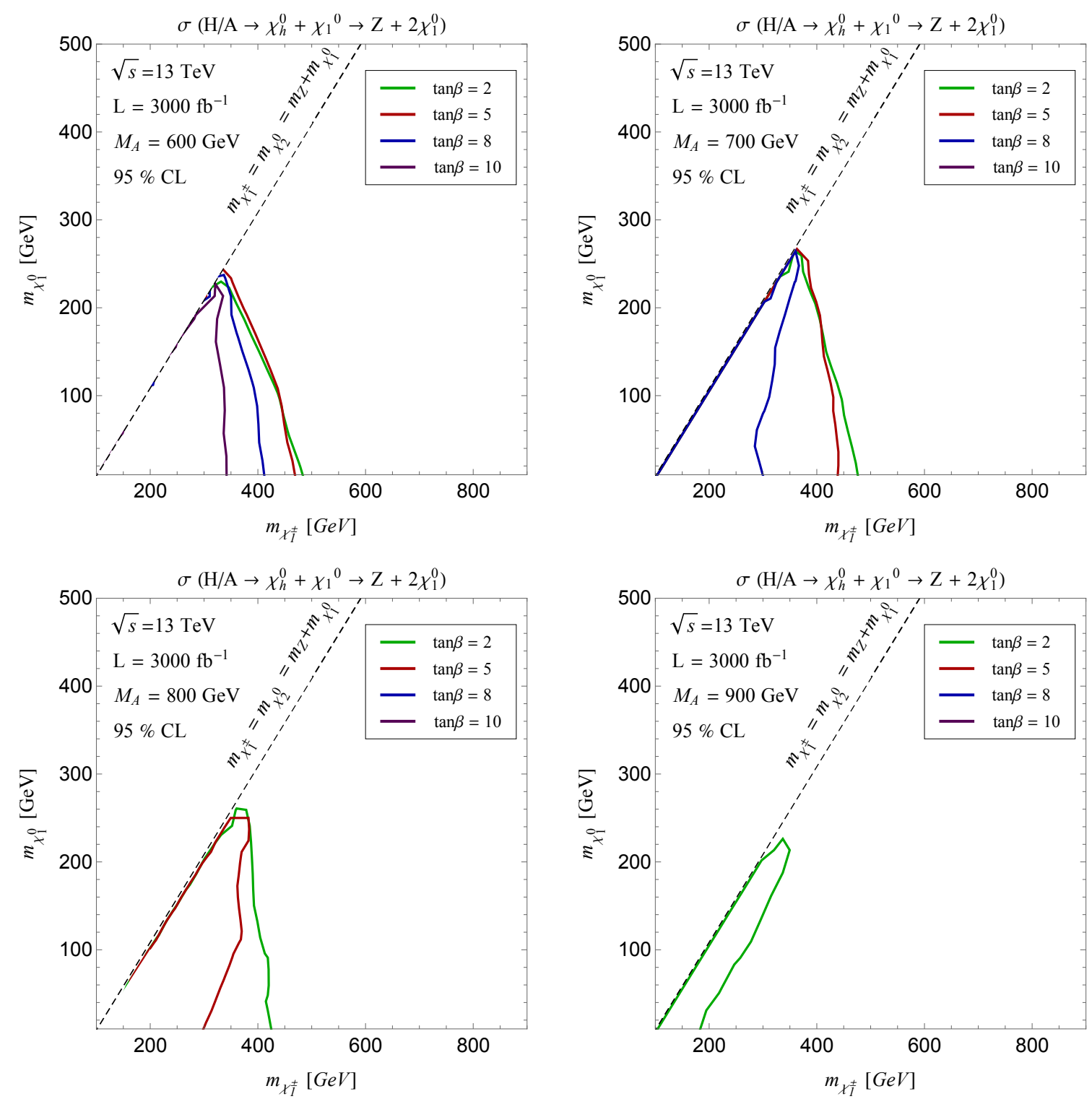

Figure 7. $95 \%$ CL bounds on the heavy neutral Higgs decay into electroweakinos $H / A \rightarrow$ $\chi_{h}^{0} \chi_{1}^{0} \rightarrow Z+2 \chi_{1}^{0}$, with $Z \rightarrow \ell^{+} \ell^{-}$. With integrated luminosity of $3000 \mathrm{fb}^{-1}$ and $m_{H / A}=$ $600,700,800,900 \mathrm{GeV}$, the sensitivities for electroweakinos are shown as contours in the $m_{\chi_{1}^{ \pm}}-m_{\chi_{1}^{0}}$ plane for $\tan \beta=2-10$.

right panel, in the case of $m_{A}=600 \mathrm{GeV}$, we see that the sensitivity is limited to low values of $\tan \beta=2$ and 5. For larger values of the Higgs masses the bounds become even weaker and vanish for $m_{A} \gtrsim 700 \mathrm{GeV}$, due to the decrease in the production cross-section $\sigma\left(g g \rightarrow H / A \rightarrow \chi_{h}^{0} \chi_{1}^{0} \rightarrow Z+2 \chi_{1}^{0}\right)$.

In figure 7 , we show the reach for $3000 \mathrm{fb}^{-1}$. We find that for heavy Higgs masses of $600-700 \mathrm{GeV}$, the reach can explore a broad range of values of $\tan \beta$. Further, for larger masses of the heavy Higgses the reach of the Higgs decay channel generally improves in the region of the kinematic limit of the $\chi_{2,3}^{0} \rightarrow Z+\chi_{1}^{0}$ decay. This is expected as the 

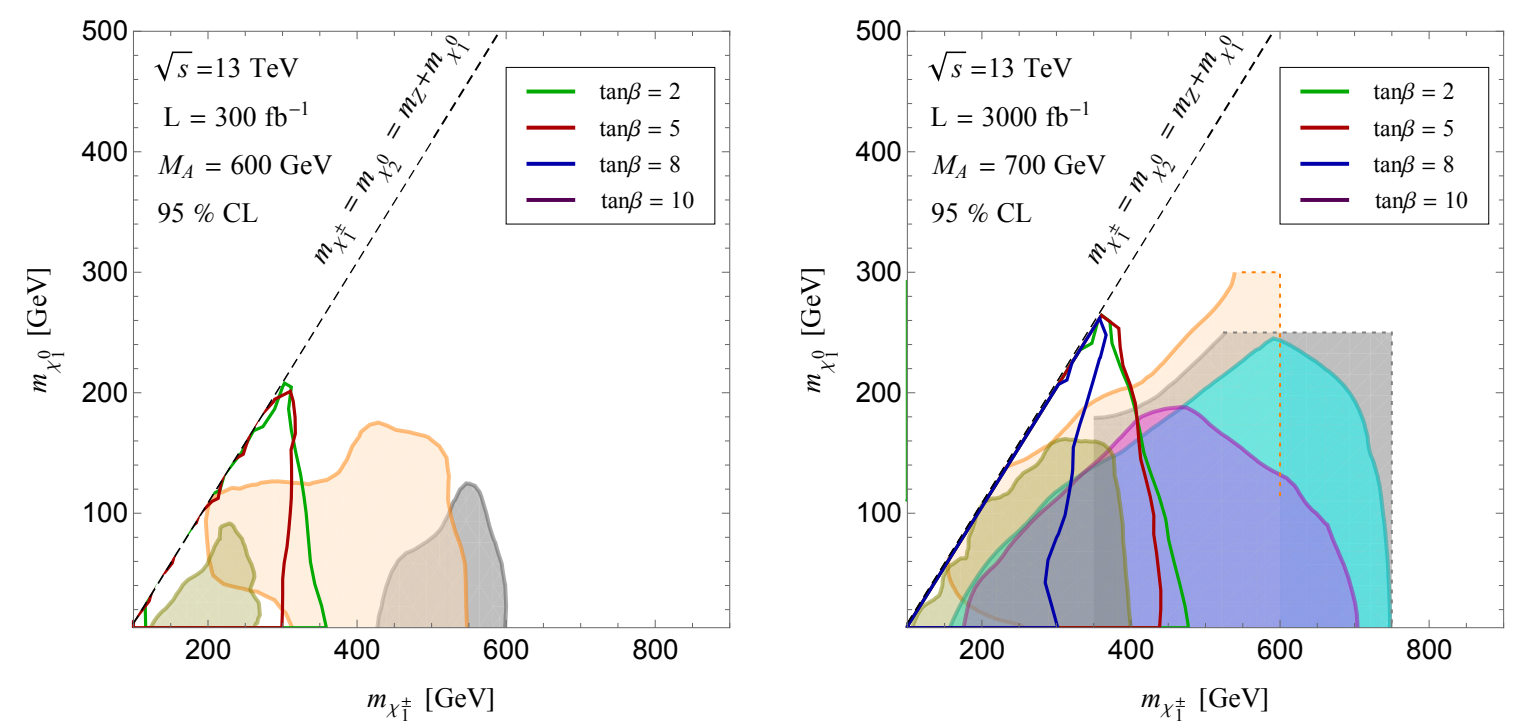

Figure 8. Comparison between the $H / A \rightarrow \chi_{h}^{0} \chi_{1}^{0} \rightarrow Z+2 \chi_{1}^{0}$ electroweakino search and the existing direct production constraints for the higgsino-like electroweakinos at HL-LHC. In the case of the resonant Higgs channel we present the bounds for $m_{A}=600$ (700) GeV for luminosity of $300(3000) \mathrm{fb}^{-1}$.

cut efficiency of the kinematics resulting from high $p_{T}$ leptons compensates the decrease in cross sections. For Higgs masses above $1 \mathrm{TeV}$, we find no reach for any values of $\tan \beta$, since beyond this point the efficiency gain cannot compete with the drop in the production cross section.

In figure 8 , we compare the $H / A \rightarrow \chi_{h}^{0} \chi_{1}^{0} \rightarrow Z+2 \chi_{1}^{0}$ search to existing direct production constraints interpreted for higgsino-like electroweakinos, as presented in the top right panel of figure 5 . The sensitivities are nicely complementary to each other. We find that there is a significant overlap in the parameter space that can be covered between the proposed Higgs decay search and the direct production searches. We note that since there is still a chance to obtain a $5 \sigma$ discovery at HL-LHC, as we show in the bottom right panel of figure 5 , the search for electroweakinos will become an alternative way of probing the existence of a heavy Higgs boson beyond the traditional decay channels to $\tau$-leptons, and top and bottom quarks. As emphasized before, the proposed Higgs decay channel also has better sensitivity close to the compressed mass region $m_{\chi_{1}^{ \pm}, \chi_{h}^{0}} \simeq m_{\chi_{1}^{0}}+Z$ compared with the direct production case, offering a unique channel to explore this region of electroweakino masses.

\section{Conclusions}

In this article we have analyzed the search for electroweakinos at the LHC, putting emphasis on the complementarity of direct and Higgs decay production modes. We have considered the case of heavy scalar superpartners and concentrated on the well motivated case of light Higgsinos and Binos. We have shown that the LHC reach in this case remains weak in 
both production channels at a luminosity of $300 \mathrm{fb}^{-1}$ but becomes very promising at higher luminosities.

In the direct production mode, the high luminosity LHC, with a total integrated luminosity of $3000 \mathrm{fb}^{-1}$ will be able to cover up to masses of the lightest chargino and second and third lightest neutralino states up to the order of $800 \mathrm{GeV}$ for lightest neutralinos lower than about $300 \mathrm{GeV}$. This is comparable to the current reach at the LHC for the case of light Winos and significantly larger than the reach for light Higgsinos at $300 \mathrm{fb}^{-1}$, that go up to chargino masses of about $600 \mathrm{GeV}$ for lightest neutralinos not heavier than $150 \mathrm{GeV}$. In spite of the considerable reach, a large gap is open when the mass differences between the heavier electroweakino states and the lightest neutralino state become small.

The reach in the heavy Higgs decay mode depends strongly on the Higgs boson masses, and cannot go up to chargino masses as large as in the direct production mode. However, for heavy Higgs boson masses larger than $500 \mathrm{GeV}$ and smaller than about $900 \mathrm{GeV}$, and moderate values of $\tan \beta$, it can go up to chargino masses of about $500 \mathrm{GeV}$ for lightest neutralino masses not heavier than $300 \mathrm{GeV}$. Interestingly, although the coverage is not as strong as the direct production case, this search is not limited by the same kinematic considerations as the ones in the direct production mode and can cover masses up to the kinematic threshold for the decay of the heavier electroweakinos into the lightest neutralino. Moreover, this mode provides also an alternative way of looking for heavy Higgs bosons in this range of masses and hence should be a high priority for future LHC analyses.

\section{Acknowledgments}

We would like to thank S. Gori and N. Shah for very useful discussions and communication. Work at University of Chicago is supported in part by U.S. Department of Energy grant number DE-FG02-13ER41958. Work at ANL is supported in part by the U.S. Department of Energy under Contract No. DE-AC02-06CH11357. JL acknowledges support by Oehme Fellowship. During the course of this work NM has been supported by the U.S. Department of Energy, Office of Science, Office of Work- force Development for Teachers and Scientists, Office of Science Graduate Student Research (SCGSR) program. The SCGSR program is administered by the Oak Ridge Institute for Science and Education (ORISE) for the DOE. ORISE is managed by ORAU under contract number de-sc0014664.

\section{A Auxiliary figures}

In figure 4, we have shown the production cross-section for the process $H / A \rightarrow \chi_{2,3}^{0} \chi_{1}^{0} \rightarrow$ $Z+2 \chi_{1}^{0}$ for higgsino-like electroweakinos in the case of $m_{A}=600 \mathrm{GeV}$ and $\tan \beta=$ $2,5,8,10$. For reader's convenience, we also show the comparison of the production cross section for $m_{A}=600,700, \mathrm{GeV}$ and $800,900 \mathrm{GeV}$ in figure 9, and figure 10 respectively.

\section{B Results for $\operatorname{sgn}(\mu)=+1$}

In the main results presented in this paper we have assumed that $\operatorname{sgn}(\mu)=-1$. Apart from the motivations given in the introduction for this choice, it is of interest to also explore the 

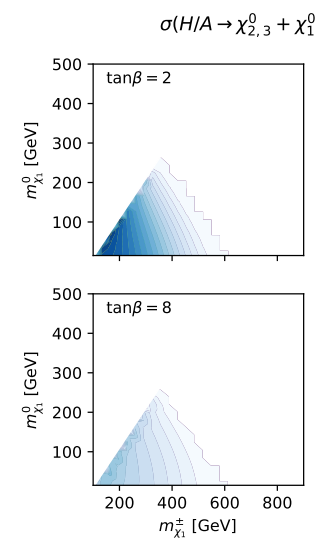
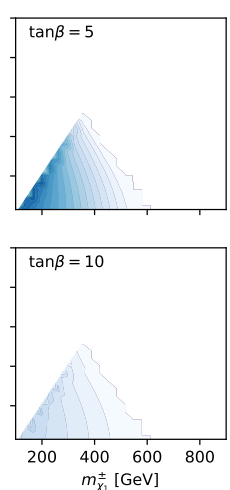
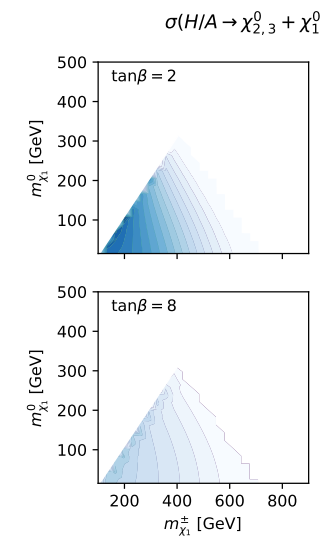
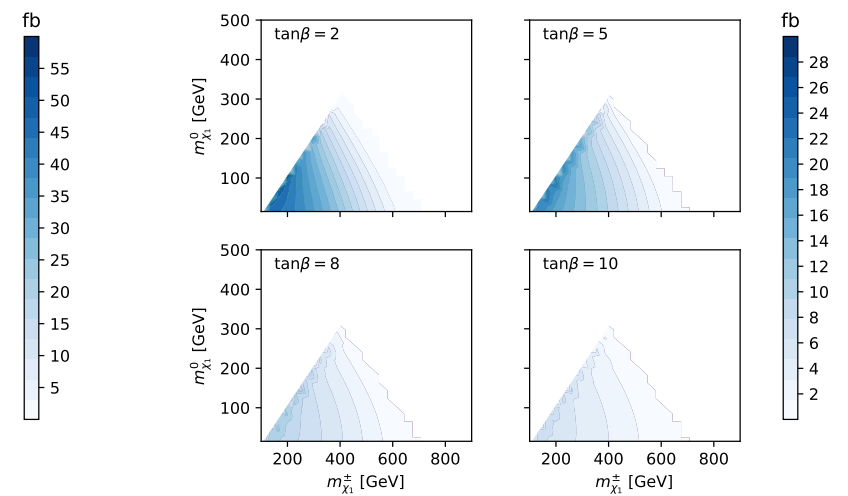

Figure 9. The production cross-section for the process $H / A \rightarrow \chi_{2,3}^{0} \chi_{1}^{0} \rightarrow Z+2 \chi_{1}^{0}$ for higgsino-like electroweakinos, with heavy scalar mass $m_{A}=600,700 \mathrm{GeV}$ and $\tan \beta=2,5,8,10$.
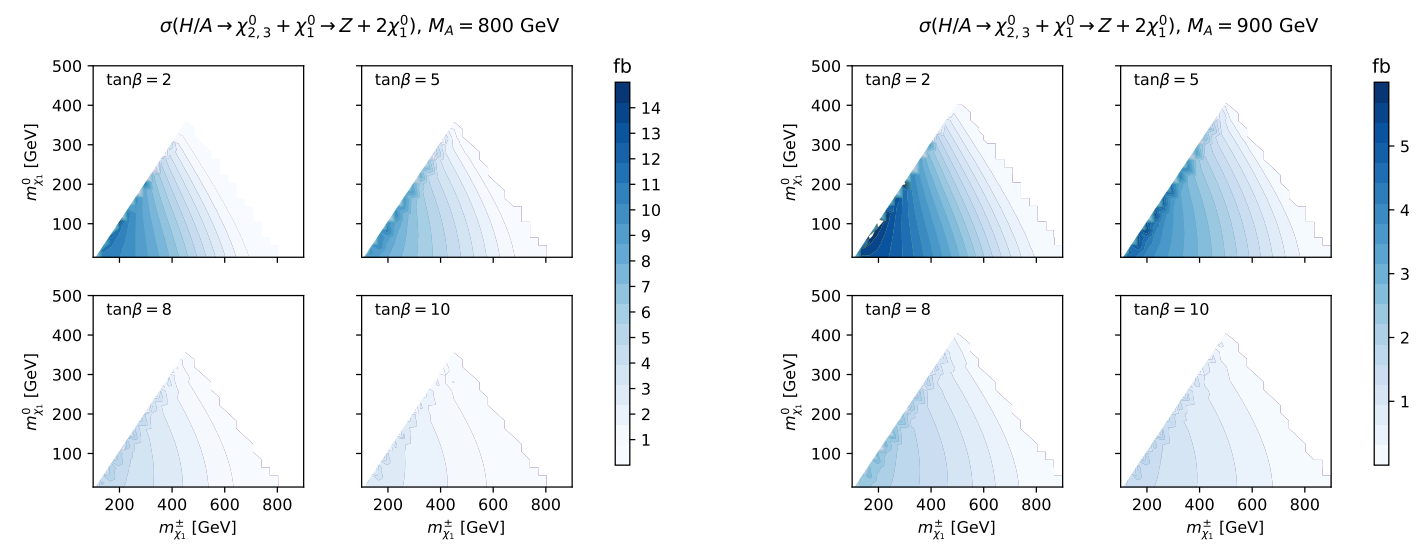

Figure 10. The production cross-section for the process $H / A \rightarrow \chi_{2,3}^{0} \chi_{1}^{0} \rightarrow Z+2 \chi_{1}^{0}$ for higgsino-like electroweakinos, with heavy scalar mass $m_{A}=800,900 \mathrm{GeV}$ and $\tan \beta=2,5,8,10$.

possibility of $\operatorname{sgn}(\mu)=+1$. We have studied the corresponding production cross sections in this case and find only minor qualitative differences to the main results. However, for completeness in figure 11 we show the comparison between $H / A \rightarrow \chi_{h}^{0} \chi_{1}^{0} \rightarrow Z+2 \chi_{1}^{0}$ electroweakino search and the existing direct production constraints for the higgsino-like electroweakinos at HL-LHC assuming $\operatorname{sgn}(\mu)=+1$. In the case of the resonant Higgs channel we present the bounds for $m_{A}=600(700) \mathrm{GeV}$ for $300(3000) \mathrm{fb}^{-1}$ luminosity.

Open Access. This article is distributed under the terms of the Creative Commons Attribution License (CC-BY 4.0), which permits any use, distribution and reproduction in any medium, provided the original author(s) and source are credited. 

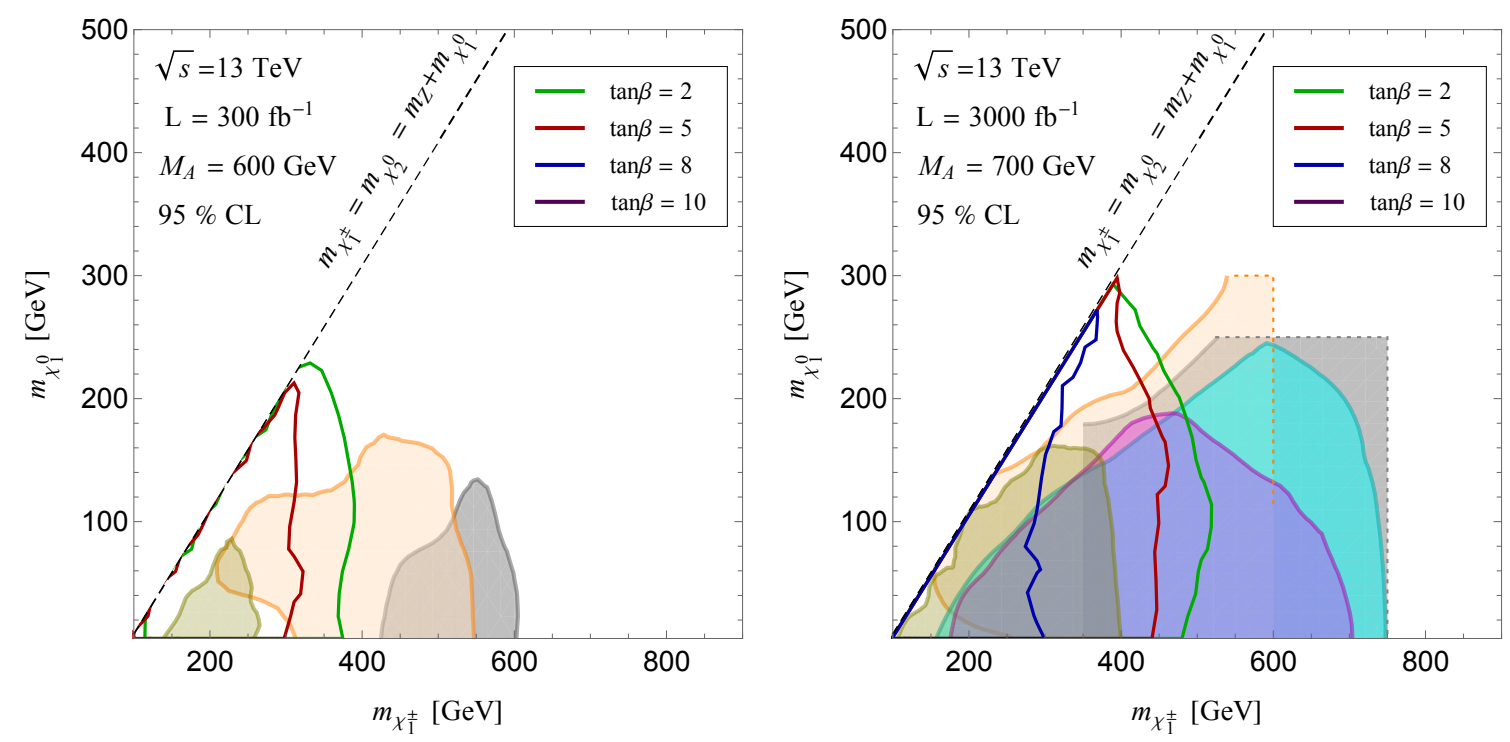

Figure 11. Comparison between the $H / A \rightarrow \chi_{h}^{0} \chi_{1}^{0} \rightarrow Z+2 \chi_{1}^{0}$ electroweakino search and the existing direct production constraints for the higgsino-like electroweakinos at HL-LHC assuming $\operatorname{sgn}(\mu)=+1$. In the case of the resonant Higgs channel we present the bounds for $m_{A}=600(700) \mathrm{GeV}$ for luminosity of $300(3000) \mathrm{fb}^{-1}$.

\section{References}

[1] ATLAS collaboration, Search for squarks and gluinos in final states with jets and missing transverse momentum using $36 \mathrm{fb}^{-1}$ of $\sqrt{s}=13 \mathrm{TeV}$ pp collision data with the ATLAS detector, Phys. Rev. D 97 (2018) 112001 [arXiv:1712.02332] [INSPIRE].

[2] CMS collaboration, Search for natural and split supersymmetry in proton-proton collisions at $\sqrt{s}=13 \mathrm{TeV}$ in final states with jets and missing transverse momentum, JHEP 05 (2018) 025 [arXiv: 1802.02110] [inSPIRE].

[3] CMS collaboration, Search for electroweak production of charginos and neutralinos in WH events in proton-proton collisions at $\sqrt{s}=13$ TeV, JHEP 11 (2017) 029 [arXiv: 1706. 09933] [INSPIRE].

[4] CMS collaboration, Search for electroweak production of charginos and neutralinos in multilepton final states in proton-proton collisions at $\sqrt{s}=13 \mathrm{TeV}$, JHEP 03 (2018) 166 [arXiv: 1709.05406] [INSPIRE].

[5] CMS collaboration, Combined search for electroweak production of charginos and neutralinos in proton-proton collisions at $\sqrt{s}=13$ TeV, JHEP 03 (2018) 160 [arXiv: 1801.03957] [INSPIRE].

[6] CMS collaboration, Search for new physics in events with two soft oppositely charged leptons and missing transverse momentum in proton-proton collisions at $\sqrt{s}=13 \mathrm{TeV}$, Phys. Lett. B 782 (2018) 440 [arXiv:1801.01846] [INSPIRE].

[7] ATLAS collaboration, Search for electroweak production of supersymmetric particles in final states with two or three leptons at $\sqrt{s}=13 \mathrm{TeV}$ with the ATLAS detector, Eur. Phys. J. $C 78$ (2018) 995 [arXiv: 1803.02762] [INSPIRE]. 
[8] ATLAS collaboration, Search for chargino-neutralino production using recursive jigsaw reconstruction in final states with two or three charged leptons in proton-proton collisions at $\sqrt{s}=13$ TeV with the ATLAS detector, Phys. Rev. D 98 (2018) 092012 [arXiv: 1806. 02293] [INSPIRE].

[9] ATLAS collaboration, Search for chargino and neutralino production in final states with a Higgs boson and missing transverse momentum at $\sqrt{s}=13 \mathrm{TeV}$ with the ATLAS detector, Phys. Rev. D 100 (2019) 012006 [arXiv: 1812.09432] [INSPIRE].

[10] ATLAS collaboration, Search for direct production of electroweakinos in final states with one lepton, missing transverse momentum and a Higgs boson decaying into two b-jets in pp collisions at $\sqrt{s}=13 \mathrm{TeV}$ with the ATLAS detector, Eur. Phys. J. C 80 (2020) 691 [arXiv: 1909.09226] [INSPIRE].

[11] ATLAS collaboration, Search for electroweak production of charginos and sleptons decaying in final states with two leptons and missing transverse momentum in $\sqrt{s}=13 \mathrm{TeV} p p$ collisions using the ATLAS detector, Tech. Rep. ATLAS-CONF-2019-008, CERN, Geneva, Switzerland (2019).

[12] ATLAS collaboration, Search for chargino-neutralino production with mass splittings near the electroweak scale in three-lepton final states in $\sqrt{s}=13 \mathrm{TeV}$ pp collisions with the ATLAS detector, Tech. Rep. ATLAS-CONF-2019-020, CERN, Geneva, Switzerland (2019).

[13] ATLAS collaboration, Search for direct production of electroweakinos in final states with missing transverse momentum and a Higgs boson decaying into photons in pp collisions at $\sqrt{s}=13 \mathrm{TeV}$ with the ATLAS detector, arXiv:2004.10894 [INSPIRE].

[14] P. Fayet, Supersymmetry and weak, electromagnetic and strong interactions, Phys. Lett. B 64 (1976) 159 [INSPIRE].

[15] P. Fayet, Spontaneously broken supersymmetric theories of weak, electromagnetic and strong interactions, Phys. Lett. B 69 (1977) 489 [INSPIRE].

[16] J.F. Gunion et al., Calculation and phenomenology of two-body decays of neutralinos and charginos to W, Z, and Higgs bosons, Int. J. Mod. Phys. A 2 (1987) 1145 [INSPIRE].

[17] H.E. Haber and G.L. Kane, The search for supersymmetry: probing physics beyond the Standard Model, Phys. Rept. 117 (1985) 75 [INSPIRE].

[18] S.P. Martin, A supersymmetry primer, Adv. Ser. Direct. High Energy Phys. 18 (1998) 1 [Adv. Ser. Direct. High Energy Phys. 21 (2010) 1] [hep-ph/9709356] [INSPIRE].

[19] R. Barbieri and G.F. Giudice, Upper bounds on supersymmetric particle masses, Nucl. Phys. B 306 (1988) 63 [INSPIRE].

[20] B. de Carlos and J.A. Casas, One loop analysis of the electroweak breaking in supersymmetric models and the fine tuning problem, Phys. Lett. B 309 (1993) 320 [hep-ph/9303291] [INSPIRE].

[21] C. Brust, A. Katz, S. Lawrence and R. Sundrum, SUSY, the third generation and the LHC, JHEP 03 (2012) 103 [arXiv:1110.6670] [INSPIRE].

[22] H. Goldberg, Constraint on the photino mass from cosmology, Phys. Rev. Lett. 50 (1983) 1419 [Erratum ibid. 103 (2009) 099905] [INSPIRE].

[23] J.R. Ellis, J.S. Hagelin, D.V. Nanopoulos, K.A. Olive and M. Srednicki, Supersymmetric relics from the big bang, Nucl. Phys. B 238 (1984) 453 [inSPIRE]. 
[24] F. Moortgat, S. Abdullin and D. Denegri, Observability of MSSM Higgs bosons via sparticle decay modes in CMS, hep-ph/0112046 [INSPIRE].

[25] D. Denegri et al., Summary of the CMS discovery potential for the MSSM SUSY Higgses, hep-ph/0112045 [INSPIRE].

[26] F. Moortgat, Observability of MSSM Higgs bosons decaying to sparticles at the LHC, in $36^{\text {th }}$ Rencontres de Moriond on QCD and hadronic interactions, (2001), pg. 83 [hep-ph/0105081] [INSPIRE].

[27] CMS collaboration, CMS technical design report, volume II: physics performance, J. Phys. G 34 (2007) 995 [INSPIRE].

[28] E. Arganda, J. Diaz-Cruz and A. Szynkman, Decays of $H^{0} / A^{0}$ in supersymmetric scenarios with heavy sfermions, Eur. Phys. J. C 73 (2013) 2384 [arXiv:1211.0163] [InSPIRE].

[29] T. Han, Z. Liu and A. Natarajan, Dark matter and Higgs bosons in the MSSM, JHEP 11 (2013) 008 [arXiv: 1303.3040] [INSPIRE].

[30] H. Baer et al., Radiatively-driven natural supersymmetry at the LHC, JHEP 12 (2013) 013 [Erratum ibid. 06 (2015) 053] [arXiv:1310.4858] [INSPIRE].

[31] H. Baer, V. Barger, P. Huang, D. Mickelson, M. Padeffke-Kirkland and X. Tata, Natural SUSY with a bino- or wino-like LSP, Phys. Rev. D 91 (2015) 075005 [arXiv:1501.06357] [INSPIRE].

[32] N. Craig, F. D'Eramo, P. Draper, S. Thomas and H. Zhang, The hunt for the rest of the Higgs bosons, JHEP 06 (2015) 137 [arXiv:1504.04630] [INSPIRE].

[33] R.K. Barman, B. Bhattacherjee, A. Chakraborty and A. Choudhury, Study of MSSM heavy Higgs bosons decaying into charginos and neutralinos, Phys. Rev. D 94 (2016) 075013 [arXiv: 1607.00676] [INSPIRE].

[34] S. Kulkarni and L. Lechner, Characterizing simplified models for heavy Higgs decays to supersymmetric particles, arXiv:1711.00056 [INSPIRE].

[35] G.H. Duan, W. Wang, L. Wu, J.M. Yang and J. Zhao, Probing GeV-scale MSSM neutralino dark matter in collider and direct detection experiments, Phys. Lett. B 778 (2018) 296 [arXiv: 1711.03893] [INSPIRE].

[36] A.D. Medina and M.A. Schmidt, Enlarging regions of the MSSM parameter space for large $\tan \beta$ via SUSY decays of the heavy Higgs bosons, JHEP 08 (2017) 095 [arXiv: 1706. 04994] [INSPIRE].

[37] E. Arganda, V. Martin-Lozano, A.D. Medina and N. Mileo, Potential discovery of staus through heavy Higgs boson decays at the LHC, JHEP 09 (2018) 056 [arXiv: 1804.10698] [INSPIRE].

[38] E. Bagnaschi et al., MSSM Higgs boson searches at the LHC: benchmark scenarios for run 2 and beyond, Eur. Phys. J. C 79 (2019) 617 [arXiv: 1808.07542] [INSPIRE].

[39] S. Gori, Z. Liu and B. Shakya, Heavy Higgs as a portal to the supersymmetric electroweak sector, JHEP 04 (2019) 049 [arXiv:1811.11918] [INSPIRE].

[40] H. Bahl, S. Liebler and T. Stefaniak, MSSM Higgs benchmark scenarios for run 2 and beyond: the low $\tan \beta$ region, Eur. Phys. J. C 79 (2019) 279 [arXiv:1901. 05933] [InSPIRE].

[41] S. Alipour-Fard, N. Craig, S. Gori, S. Koren and D. Redigolo, The second Higgs at the lifetime frontier, JHEP 07 (2020) 029 [arXiv: 1812.09315] [INSPIRE]. 
[42] A. Canepa, T. Han and X. Wang, The search for electroweakinos, arXiv: 2003.05450 [INSPIRE].

[43] A. Adhikary, B. Bhattacherjee, R.M. Godbole, N. Khan and S. Kulkarni, Searching for heavy Higgs in supersymmetric final states at the LHC, arXiv:2002.07137 [INSPIRE].

[44] N.D. Christensen, T. Han, Z. Liu and S. Su, Low-mass Higgs bosons in the NMSSM and their LHC implications, JHEP 08 (2013) 019 [arXiv: 1303.2113] [INSPIRE].

[45] B. Dutta, Y. Gao and B. Shakya, Light Higgsino decays as a probe of the NMSSM, Phys. Rev. D 91 (2015) 035016 [arXiv:1412.2774] [inSPIRE].

[46] T. Han, Z. Liu and S. Su, Light neutralino dark matter: direct/indirect detection and collider searches, JHEP 08 (2014) 093 [arXiv:1406.1181] [INSPIRE].

[47] F. Wang, W. Wang, L. Wu, J.M. Yang and M. Zhang, Probing degenerate heavy Higgs bosons in NMSSM with vector-like particles, Int. J. Mod. Phys. A 32 (2017) 1745005 [arXiv: 1512.08434] [INSPIRE].

[48] U. Ellwanger and M. Rodriguez-Vazquez, Simultaneous search for extra light and heavy Higgs bosons via cascade decays, JHEP 11 (2017) 008 [arXiv:1707.08522] [INSPIRE].

[49] S. Baum, K. Freese, N.R. Shah and B. Shakya, NMSSM Higgs boson search strategies at the LHC and the mono-Higgs signature in particular, Phys. Rev. D 95 (2017) 115036 [arXiv: 1703.07800] [INSPIRE].

[50] S. Baum, N.R. Shah and K. Freese, The NMSSM is within reach of the LHC: mass correlations \& decay signatures, JHEP 04 (2019) 011 [arXiv:1901.02332] [INSPIRE].

[51] P. Draper, G. Lee and C.E.M. Wagner, Precise estimates of the Higgs mass in heavy supersymmetry, Phys. Rev. D 89 (2014) 055023 [arXiv: 1312.5743] [INSPIRE].

[52] E. Bagnaschi, G.F. Giudice, P. Slavich and A. Strumia, Higgs mass and unnatural supersymmetry, JHEP 09 (2014) 092 [arXiv: 1407.4081] [INSPIRE].

[53] J. Pardo Vega and G. Villadoro, SusyHD: Higgs mass determination in supersymmetry, JHEP 07 (2015) 159 [arXiv:1504.05200] [INSPIRE].

[54] G. Lee and C.E.M. Wagner, Higgs bosons in heavy supersymmetry with an intermediate $m_{A}$, Phys. Rev. D 92 (2015) 075032 [arXiv:1508.00576] [inSPIRE].

[55] H. Bahl, S. Heinemeyer, W. Hollik and G. Weiglein, Reconciling EFT and hybrid calculations of the light MSSM Higgs-boson mass, Eur. Phys. J. C 78 (2018) 57 [arXiv: 1706. 00346] [INSPIRE].

[56] J.R. Ellis, J.S. Hagelin and D.V. Nanopoulos, Spin 0 leptons and the anomalous magnetic moment of the muon, Phys. Lett. B 116 (1982) 283 [INSPIRE].

[57] T. Moroi, The muon anomalous magnetic dipole moment in the minimal supersymmetric Standard Model, Phys. Rev. D 53 (1996) 6565 [Erratum ibid. 56 (1997) 4424] [hep-ph/9512396] [INSPIRE].

[58] M. Carena, G.F. Giudice and C.E.M. Wagner, Constraints on supersymmetric models from the muon anomalous magnetic moment, Phys. Lett. B 390 (1997) 234 [hep-ph/9610233] [INSPIRE].

[59] A. Czarnecki and W.J. Marciano, The muon anomalous magnetic moment: a Harbinger for 'new physics', Phys. Rev. D 64 (2001) 013014 [hep-ph/0102122] [INSPIRE]. 
[60] J.L. Feng and K.T. Matchev, Supersymmetry and the anomalous magnetic moment of the muon, Phys. Rev. Lett. 86 (2001) 3480 [hep-ph/0102146] [INSPIRE].

[61] S.P. Martin and J.D. Wells, Muon anomalous magnetic dipole moment in supersymmetric theories, Phys. Rev. D 64 (2001) 035003 [hep-ph/0103067] [INSPIRE].

[62] Muon G-2 collaboration, Final report of the muon E821 anomalous magnetic moment measurement at BNL, Phys. Rev. D 73 (2006) 072003 [hep-ex/0602035] [INSPIRE].

[63] CMS collaboration, Search for additional neutral MSSM Higgs bosons in the $\tau \tau$ final state in proton-proton collisions at $\sqrt{s}=13 \mathrm{TeV}, \mathrm{JHEP} 09$ (2018) 007 [arXiv: 1803.06553] [INSPIRE].

[64] ATLAS collaboration, Search for heavy Higgs bosons decaying into two tau leptons with the ATLAS detector using pp collisions at $\sqrt{s}=13$ TeV, Phys. Rev. Lett. 125 (2020) 051801 [arXiv: 2002.12223] [INSPIRE].

[65] N. Arkani-Hamed, A. Delgado and G.F. Giudice, The well-tempered neutralino, Nucl. Phys. B 741 (2006) 108 [hep-ph/0601041] [INSPIRE].

[66] LUX collaboration, Results from a search for dark matter in the complete LUX exposure, Phys. Rev. Lett. 118 (2017) 021303 [arXiv:1608.07648] [INSPIRE].

[67] PandaX-II collaboration, Dark matter results from 54-ton-day exposure of PandaX-II experiment, Phys. Rev. Lett. 119 (2017) 181302 [arXiv:1708.06917] [INSPIRE].

[68] XENON collaboration, First dark matter search results from the XENON1T experiment, Phys. Rev. Lett. 119 (2017) 181301 [arXiv:1705.06655] [INSPIRE].

[69] XENON collaboration, Dark matter search results from a one ton-year exposure of XENON1 T, Phys. Rev. Lett. 121 (2018) 111302 [arXiv:1805.12562] [INSPIRE].

[70] H. Baer, V. Barger and H. Serce, SUSY under siege from direct and indirect WIMP detection experiments, Phys. Rev. D 94 (2016) 115019 [arXiv:1609.06735] [InSPIRE].

[71] P. Huang, R.A. Roglans, D.D. Spiegel, Y. Sun and C.E.M. Wagner, Constraints on supersymmetric dark matter for heavy scalar superpartners, Phys. Rev. D 95 (2017) 095021 [arXiv: 1701.02737] [INSPIRE].

[72] G. Gelmini, P. Gondolo, A. Soldatenko and C.E. Yaguna, The effect of a late decaying scalar on the neutralino relic density, Phys. Rev. D 74 (2006) 083514 [hep-ph/0605016] [InSPIRE].

[73] J.R. Ellis, T. Falk, K.A. Olive and M. Srednicki, Calculations of neutralino-stau coannihilation channels and the cosmologically relevant region of MSSM parameter space, Astropart. Phys. 13 (2000) 181 [Erratum ibid. 15 (2001) 413] [hep-ph/9905481] [INSPIRE].

[74] T. Han, F. Kling, S. Su and Y. Wu, Unblinding the dark matter blind spots, JHEP 02 (2017) 057 [arXiv: 1612.02387] [INSPIRE].

[75] M. Carena, J. Osborne, N.R. Shah and C.E.M. Wagner, Supersymmetry and LHC missing energy signals, Phys. Rev. D 98 (2018) 115010 [arXiv:1809.11082] [INSPIRE].

[76] M. Carena, J. Osborne, N.R. Shah and C.E.M. Wagner, Return of the WIMP: missing energy signals and the galactic center excess, Phys. Rev. D 100 (2019) 055002 [arXiv: 1905.03768] [INSPIRE].

[77] H. Baer, A. Mustafayev, E.-K. Park and X. Tata, Collider signals and neutralino dark matter detection in relic-density-consistent models without universality, JHEP 05 (2008) 058 [arXiv: 0802.3384] [INSPIRE]. 
[78] P. Huang and C.E.M. Wagner, Blind spots for neutralino dark matter in the MSSM with an intermediate $m_{A}$, Phys. Rev. D 90 (2014) 015018 [arXiv: 1404.0392] [INSPIRE].

[79] M. Abdughani, L. Wu and J.M. Yang, Status and prospects of light bino-higgsino dark matter in natural SUSY, Eur. Phys. J. C 78 (2018) 4 [arXiv:1705.09164] [InSPIRE].

[80] S. Baum, M. Carena, N.R. Shah and C.E.M. Wagner, Higgs portals for thermal dark matter. EFT perspectives and the NMSSM, JHEP 04 (2018) 069 [arXiv:1712.09873] [INSPIRE].

[81] M. Endo, K. Hamaguchi, S. Iwamoto and K. Yanagi, Probing minimal SUSY scenarios in the light of muon $g-2$ and dark matter, JHEP 06 (2017) 031 [arXiv:1704.05287] [INSPIRE].

[82] T. Han, H. Liu, S. Mukhopadhyay and X. Wang, Dark matter blind spots at one-loop, JHEP 03 (2019) 080 [arXiv:1810.04679] [INSPIRE].

[83] J.R. Ellis, A. Ferstl and K.A. Olive, Reevaluation of the elastic scattering of supersymmetric dark matter, Phys. Lett. B 481 (2000) 304 [hep-ph/0001005] [INSPIRE].

[84] E. Bagnaschi et al., Likelihood analysis of the pMSSM11 in light of LHC $13 \mathrm{TeV}$ data, Eur. Phys. J. C 78 (2018) 256 [arXiv:1710.11091] [INSPIRE].

[85] J. Cao, L. Meng, Y. Yue, H. Zhou and P. Zhu, Suppressing the scattering of WIMP dark matter and nucleons in supersymmetric theories, Phys. Rev. D 101 (2020) 075003 [arXiv: 1910.14317] [INSPIRE].

[86] W. Porod, SPheno, a program for calculating supersymmetric spectra, SUSY particle decays and SUSY particle production at $e^{+} e^{-}$colliders, Comput. Phys. Commun. 153 (2003) 275 [hep-ph/0301101] [INSPIRE].

[87] W. Porod and F. Staub, SPheno 3.1: extensions including flavour, CP-phases and models beyond the MSSM, Comput. Phys. Commun. 183 (2012) 2458 [arXiv:1104.1573] [INSPIRE].

[88] H. Bahl et al., Precision calculations in the MSSM Higgs-boson sector with FeynHiggs 2.14, Comput. Phys. Commun. 249 (2020) 107099 [arXiv: 1811.09073] [InSPIRE].

[89] H. Bahl and W. Hollik, Precise prediction for the light MSSM Higgs boson mass combining effective field theory and fixed-order calculations, Eur. Phys. J. C 76 (2016) 499 [arXiv: 1608.01880] [INSPIRE].

[90] T. Hahn, S. Heinemeyer, W. Hollik, H. Rzehak and G. Weiglein, High-precision predictions for the light CP-even Higgs boson mass of the minimal supersymmetric Standard Model, Phys. Rev. Lett. 112 (2014) 141801 [arXiv:1312.4937] [INSPIRE].

[91] W. Hollik and S. Paßehr, Higgs boson masses and mixings in the complex MSSM with two-loop top-Yukawa-coupling corrections, JHEP 10 (2014) 171 [arXiv:1409.1687] [INSPIRE].

[92] G. Degrassi, S. Heinemeyer, W. Hollik, P. Slavich and G. Weiglein, Towards high precision predictions for the MSSM Higgs sector, Eur. Phys. J. C 28 (2003) 133 [hep-ph/0212020] [INSPIRE].

[93] S. Heinemeyer, W. Hollik and G. Weiglein, The masses of the neutral CP-even Higgs bosons in the MSSM: accurate analysis at the two loop level, Eur. Phys. J. C 9 (1999) 343 [hep-ph/9812472] [INSPIRE]. 
[94] S. Heinemeyer, W. Hollik and G. Weiglein, FeynHiggs: a program for the calculation of the masses of the neutral CP even Higgs bosons in the MSSM, Comput. Phys. Commun. 124 (2000) 76 [hep-ph/9812320] [INSPIRE].

[95] A. Djouadi, M.M. Muhlleitner and M. Spira, Decays of supersymmetric particles: the program SUSY-HIT (SUspect-SdecaY-HDECAY-InTerface), Acta Phys. Polon. B 38 (2007) 635 [hep-ph/0609292] [INSPIRE].

[96] T. Han, S. Padhi and S. Su, Electroweakinos in the light of the Higgs boson, Phys. Rev. D 88 (2013) 115010 [arXiv:1309.5966] [inSPIRE].

[97] J. Alwall et al., The automated computation of tree-level and next-to-leading order differential cross sections, and their matching to parton shower simulations, JHEP $\mathbf{0 7}$ (2014) 079 [arXiv: 1405.0301] [InSPIRE].

[98] W. Beenakker, R. Hopker and M. Spira, PROSPINO: a program for the production of supersymmetric particles in next-to-leading order QCD, hep-ph/9611232 [INSPIRE].

[99] W. Beenakker, M. Klasen, M. Krämer, T. Plehn, M. Spira and P.M. Zerwas, The production of charginos/neutralinos and sleptons at hadron colliders, Phys. Rev. Lett. 83 (1999) 3780 [Erratum ibid. 100 (2008) 029901] [hep-ph/9906298] [INSPIRE].

[100] ATLAS collaboration, Physics at a high-luminosity LHC with ATLAS, arXiv:1307.7292 [INSPIRE].

[101] CMS collaboration, Projected performance of an upgraded CMS detector at the LHC and HL-LHC: contribution to the Snowmass process, in Community summer study 2013: Snowmass on the Mississippi, (2013) [arXiv:1307.7135] [INSPIRE].

[102] M. Carena, S. Mrenna and C.E.M. Wagner, MSSM Higgs boson phenomenology at the Tevatron collider, Phys. Rev. D 60 (1999) 075010 [hep-ph/9808312] [INSPIRE].

[103] M. Carena and Z. Liu, Challenges and opportunities for heavy scalar searches in the $t \bar{t}$ channel at the LHC, JHEP 11 (2016) 159 [arXiv: 1608.07282] [INSPIRE].

[104] R. Hempfling, Yukawa coupling unification with supersymmetric threshold corrections, Phys. Rev. D 49 (1994) 6168 [INSPIRE].

[105] L.J. Hall, R. Rattazzi and U. Sarid, The top quark mass in supersymmetric $\mathrm{SO}(10)$ unification, Phys. Rev. D 50 (1994) 7048 [hep-ph/9306309] [INSPIRE].

[106] M. Carena, M. Olechowski, S. Pokorski and C.E.M. Wagner, Electroweak symmetry breaking and bottom-top Yukawa unification, Nucl. Phys. B 426 (1994) 269 [hep-ph/9402253] [INSPIRE].

[107] M. Carena, H.E. Haber, I. Low, N.R. Shah and C.E.M. Wagner, Complementarity between nonstandard Higgs boson searches and precision Higgs boson measurements in the MSSM, Phys. Rev. D 91 (2015) 035003 [arXiv:1410.4969] [INSPIRE].

[108] H.E. Haber, S. Heinemeyer and T. Stefaniak, The impact of two-loop effects on the scenario of MSSM Higgs alignment without decoupling, Eur. Phys. J. C 77 (2017) 742 [arXiv: 1708.04416] [INSPIRE].

[109] M. Carena, H.E. Haber, I. Low, N.R. Shah and C.E.M. Wagner, Alignment limit of the NMSSM Higgs sector, Phys. Rev. D 93 (2016) 035013 [arXiv: 1510.09137] [INSPIRE].

[110] K. Benakli, M.D. Goodsell and S.L. Williamson, Higgs alignment from extended supersymmetry, Eur. Phys. J. C 78 (2018) 658 [arXiv:1801.08849] [INSPIRE]. 
[111] N.M. Coyle and C.E.M. Wagner, Dynamical Higgs field alignment in the NMSSM, Phys. Rev. D 101 (2020) 055037 [arXiv: 1912.01036] [INSPIRE].

[112] H. Bahl, P. Bechtle, S. Heinemeyer, S. Liebler, T. Stefaniak and G. Weiglein, HL-LHC and ILC sensitivities in the hunt for heavy Higgs bosons, arXiv:2005.14536 [INSPIRE].

[113] ATLAS collaboration, Search for heavy neutral Higgs bosons produced in association with $b$-quarks and decaying into b-quarks at $\sqrt{s}=13 \mathrm{TeV}$ with the ATLAS detector, Phys. Rev. D 102 (2020) 032004 [arXiv: 1907.02749] [INSPIRE].

[114] ATLAS collaboration, Search for dijet resonances in events with an isolated charged lepton using $\sqrt{s}=13 \mathrm{TeV}$ proton-proton collision data collected by the ATLAS detector, JHEP 06 (2020) 151 [arXiv: 2002.11325] [InSPIRE].

[115] CMS collaboration, Search for heavy Higgs bosons decaying to a top quark pair in proton-proton collisions at $\sqrt{s}=13 \mathrm{TeV}$, JHEP 04 (2020) 171 [arXiv:1908.01115] [INSPIRE].

[116] ATLAS collaboration, Search for dark matter produced in association with a Higgs boson decaying to $b \bar{b}$ at $\sqrt{s}=13 \mathrm{TeV}$ with the ATLAS detector using $79.8 \mathrm{fb}^{-1}$ of proton-proton collision data, Tech. Rep. ATLAS-CONF-2018-039, CERN, Geneva, Switzerland (2018).

[117] T. Sjöstrand, S. Mrenna and P.Z. Skands, PYTHIA 6.4 physics and manual, JHEP 05 (2006) 026 [hep-ph/0603175] [INSPIRE].

[118] T. Sjöstrand et al., An introduction to PYTHIA 8.2, Comput. Phys. Commun. 191 (2015) 159 [arXiv: 1410.3012] [INSPIRE].

[119] DELPHES 3 collaboration, DELPHES 3, a modular framework for fast simulation of a generic collider experiment, JHEP 02 (2014) 057 [arXiv: 1307.6346] [INSPIRE]. 